\title{
Geochemistry and age of magmatic rocks in the unexposed Narromine, Cowal and Fairholme Igneous Complexes in the Ordovician Macquarie Arc, New South Wales
}

\author{
A. J. CRAWFORD ${ }^{1 *}$, D. R. COOKE ${ }^{1}$ AND C. M. FANNING ${ }^{2}$
}

'ARC Centre of Excellence in Ore Deposits, School of Earth Sciences, University of Tasmania, Private Bag 79, Hobart, Tas. 7001, Australia.

${ }^{2}$ PRISE, Research School of Earth Sciences, Australian National University, ACT 0200, Australia.

\begin{abstract}
Much of the northern and southern sections of the Junee-Narromine Volcanic Belt of the Ordovician Macquarie Arc in central-western New South Wales are buried beneath the sediment cover of the Great Artesian Basin. Exploration drilling of these aeromagnetically defined blocks has provided important new material to assess the temporal and magmatic affinities of the rocks in the Narromine Igneous Complex (northern end) and the Cowal and Fairholme Igneous Complexes (southern end). Basement rocks are representative of Phase 1 magmatism in the Macquarie Arc, and consist of Lower Ordovician basalts and andesites and common volcaniclastic rocks, all with high-K calc-alkaline affinities. These are very similar compositionally to the Lower Ordovician Nelungaloo Volcanics that outcrop in the central part of the Junee-Narromine Volcanic Belt west of Parkes. Intruding the basement volcanic-volcaniclastic package are three distinct igneous suites. The oldest of these, dated at ca 466-460 Ma (Middle Ordovician) and representative of Phase 2 magmatism in the Macquarie Arc, consists of stocks of monzogabbro, monzodiorite and monzonite with high-K calc-alkaline affinities, and is well represented in the Narromine and Cowal Igneous Complexes. Phase 2 suite rocks were, in turn, intruded in the Bolindian $(445 \pm 5 \mathrm{Ma})$ by kilometre-size stocks, narrow sheets and dykes made up of hornblende gabbro, diorite and granodiorite, and including quartz + plagioclase +hornblende-phyric dacitic porphyries in the Narromine Igneous Complex. These medium-K calc-alkaline rocks, assigned to the Phase 3 Copper Hill Suite that also occurs in the Molong and Rockley-Gulgong Volcanic Belts further east, crystallised from andesitic or more evolved magmas, probably derived via partial melting of low- $K$ rocks in the arc basement, or amphibolites in the subducting slab. The youngest rocks in these igneous complexes are believed to be the high-level shoshonitic intrusives in the Cowal and Fairholme Igneous Complexes, which are correlated on petrographic and compositional grounds with the Macquarie Arc Phase 4 (Bolindian and Llandovery) magmatic products best represented by the Northparkes Igneous Complex in the central part of the Junee-Narromine Volcanic Belt.
\end{abstract}

KEY WORDS: Cowal Igneous Complex, Fairholme Igneous Complex, geochemistry, geochronology, Macquarie arc, Narromine Igneous Complex.

\section{INTRODUCTION}

Geophysical data indicate that the Junee-Narromine Volcanic Belt (Figure 1) is the root section of the now disrupted Ordovician Macquarie Arc in central-western New South Wales (Direen et al. 2001). Large areas of the Junee-Narromine Volcanic Belt are buried beneath cover up to several hundred metres thick, with the only exposed section being the central part from Parkes, through the Forbes region, to the Gidginbung - Temora area (Lyons \& Wallace 1999; Raymond \& Sherwin 1999; Simpson et al. 2005; Crawford et al. 2007; Glen et al. 2007c). Much of the northern and southern extremities of this belt in New South Wales are unexposed, and are only known from exploration drilling in the Cowal and Fairholme (southern) and Narromine (northern) Igneous Complexes (Figure 1).

In this paper, we document the petrology, geochemistry and age relationships of Ordovician Macquarie Arc rocks, based on (petrographically determined) least-altered rocks sampled in drillcore from these unexposed complexes, and compare them with betterknown sections of the Junee-Narromine Volcanic Belt to contribute to a more comprehensive knowledge of the tectono-magmatic development of both this belt, and the Macquarie Arc. Figure 2 gives a summary of key magmatic units and their ages in the three major igneous complexes that make up this study,

*Corresponding author: tony.crawford@utas.edu.au

ISSN 0812-0099 print/ISSN 1440-0952 online ๑ 2007 Geological Society of Australia DOI: $10.1080 / 08120090701221714$ 

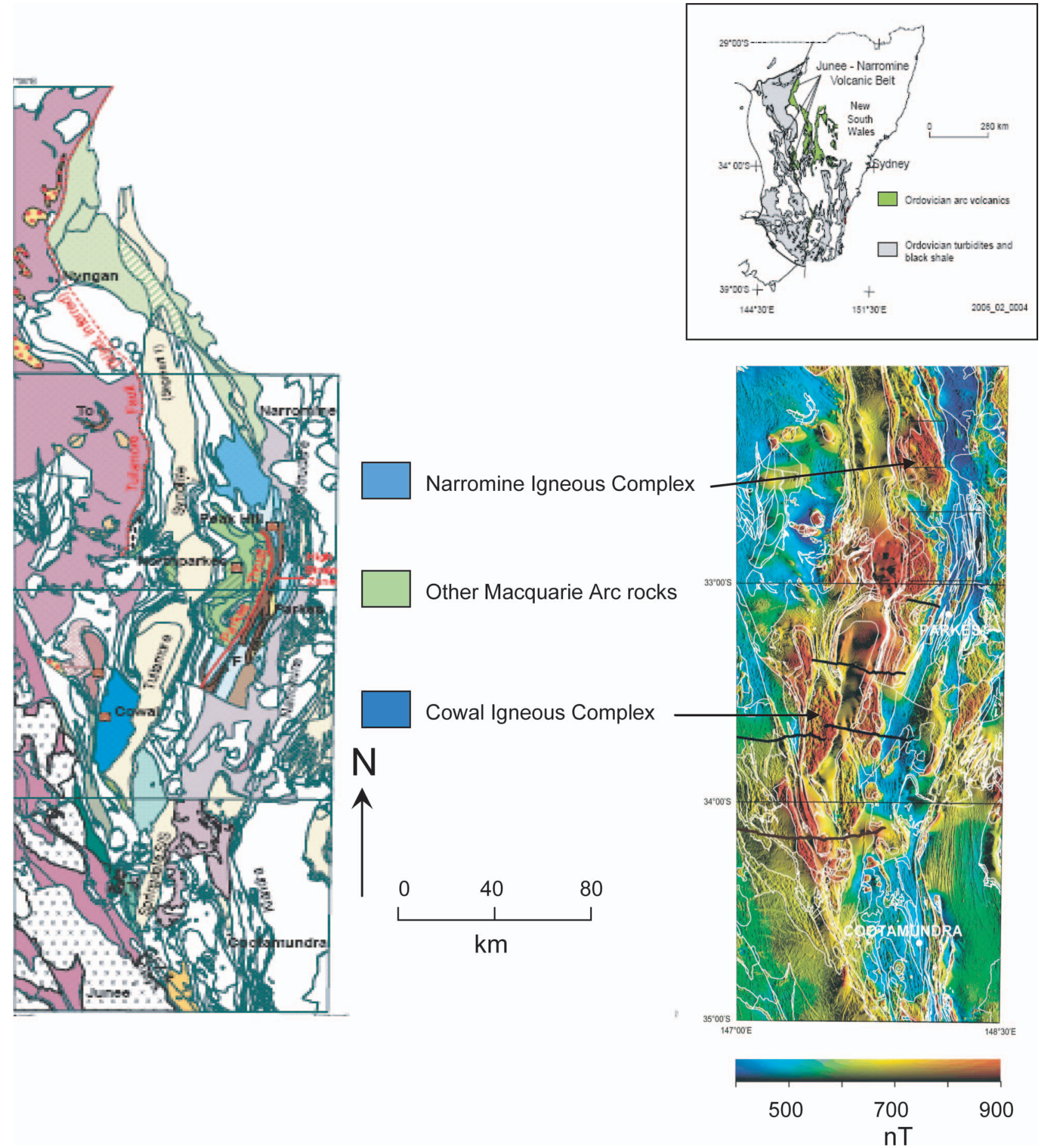

Figure 1 (a) Regional geology map showing key basement units, and (b) regional airborne magnetic map of the JuneeNarromine Volcanic Belt, showing the unexposed Narromine Igneous Complex and Cowal and Fairholme Igneous Complexes. BSZ, Booberoi Shear Zone. Inset map of New South Wales shows the location of the study region.

namely the Narromine, Cowal and Fairholme Igneous Complexes.

\section{NARROMINE IGNEOUS COMPLEX}

Outcrop of the Junee-Narromine Volcanic Belt diminishes northwards from Peak Hill towards Narromine (Sherwin 1996), and the magnetically well-defined northern part of this belt occurs entirely in the subsurface (Figure 1). The first major magnetic high in the Junee-Narromine Belt north of Peak Hill marks the $12 \times 16 \mathrm{~km}$ Narromine Igneous Complex. This complex is buried beneath $\sim 30-100 \mathrm{~m}$ of Great Artesian Basin sedimentary rocks.

Around 150 samples were examined in thin-section, and 52 freshest representative samples were analysed for this study of the Narromine Igneous Complex. 
Figure 2 Summary of the lithostratigraphy of the Narromine, Cowal and Fairholme Igneous Complexes showing major magmatic units. The Phase 4 intrusions in the Cowal and Fairholme Igneous Complexes remain undated and are assigned to Phase 4 on their basis of the pronounced shoshonitic compositions.

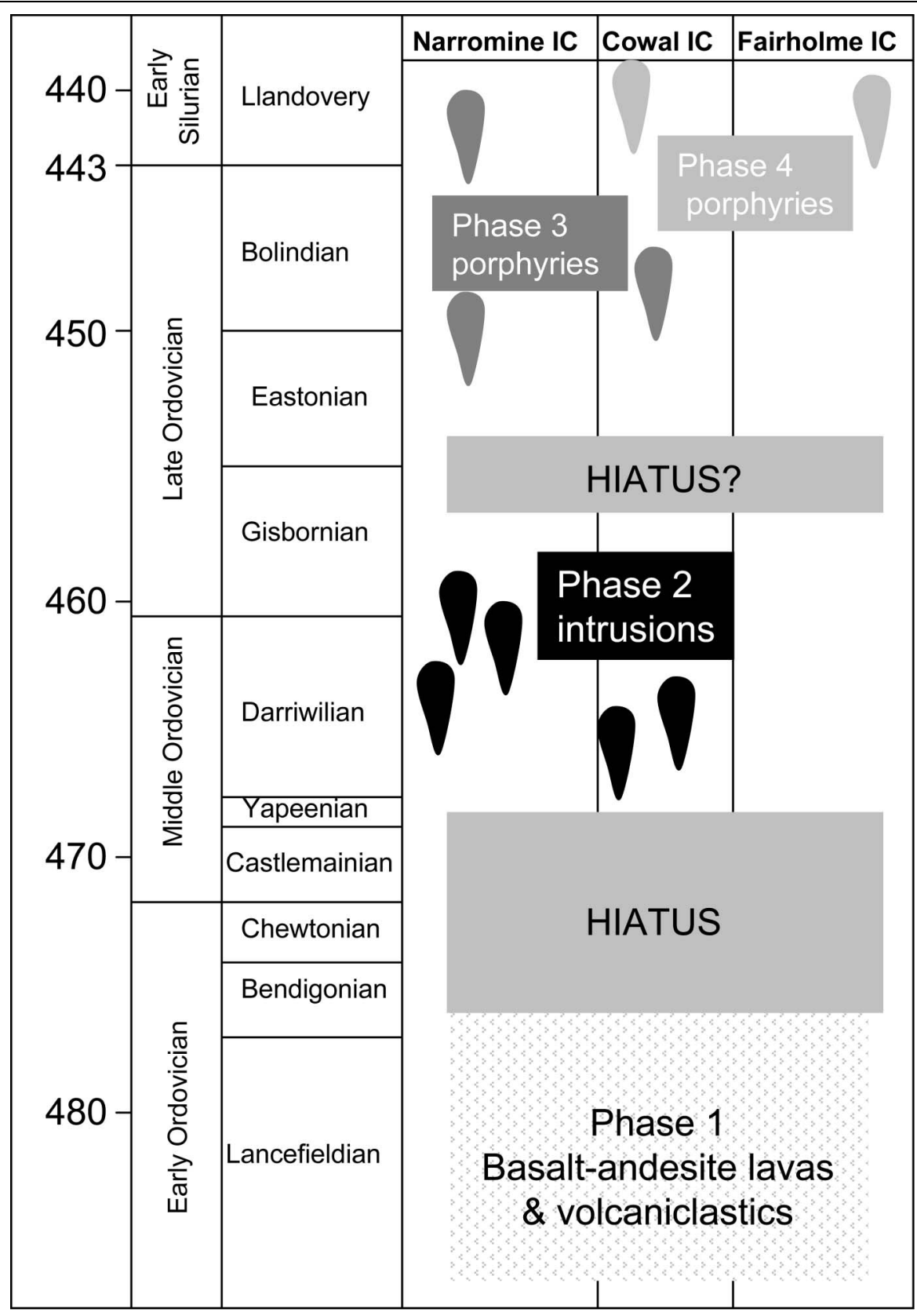

Samples were sourced from $\sim 150$ drillholes (mainly auger core with diamond tails) from a systematic grid-drilling program by Goldfields Exploration and Resolute Ltd aimed at groundtruthing a geophysical interpretation based on gravity and aeromagnetic surveys. Available data show that the Narromine Igneous Complex (Figure 3) consists of probable Ordovician basaltic and andesitic volcanics and volcaniclastic sedimentary rocks that were intruded by large plutons that vary from gabbro or monzogabbro to quartz (monzo)diorite compositions, and a later porphyritic intrusive dacite-granodiorite suite. Interpretation of available gravity and magnetic data (Glen et al. 2007d) indicates that the complex is transected by north-northwest-striking major faults (Figure 3). Interpreted Devonian sedimentary rocks border the eastern margin of the Narromine Igneous Complex, whereas Ordovician-Silurian sedimentary rocks occur along its western margins, separated from the Ordovician rocks by a near-vertical fault (Glen et al. 2007d). Most studied intrusive rocks were sampled from drillholes between $641500 \mathrm{~N}$ and $642000 \mathrm{~N}$, centred on a major intrusive complex over an across-strike distance of $\sim 15 \mathrm{~km}$ in the central part of the magnetic high (Figure 3).

\section{Volcanic and volcaniclastic rocks}

Andesitic lavas, lava breccias and volcanic conglomerates are common in the drillholes around the northern margins of the Narromine Igneous Complex, and also along its southwestern margin (Figure 3). In drillhole NACD20, plagioclase phenocryst-rich volcanic sandstones and conglomerates are intruded by dykes and sills of holocrystalline andesite to microdiorite. Most clasts (to several centimetres long) in the conglomerates 
(a)

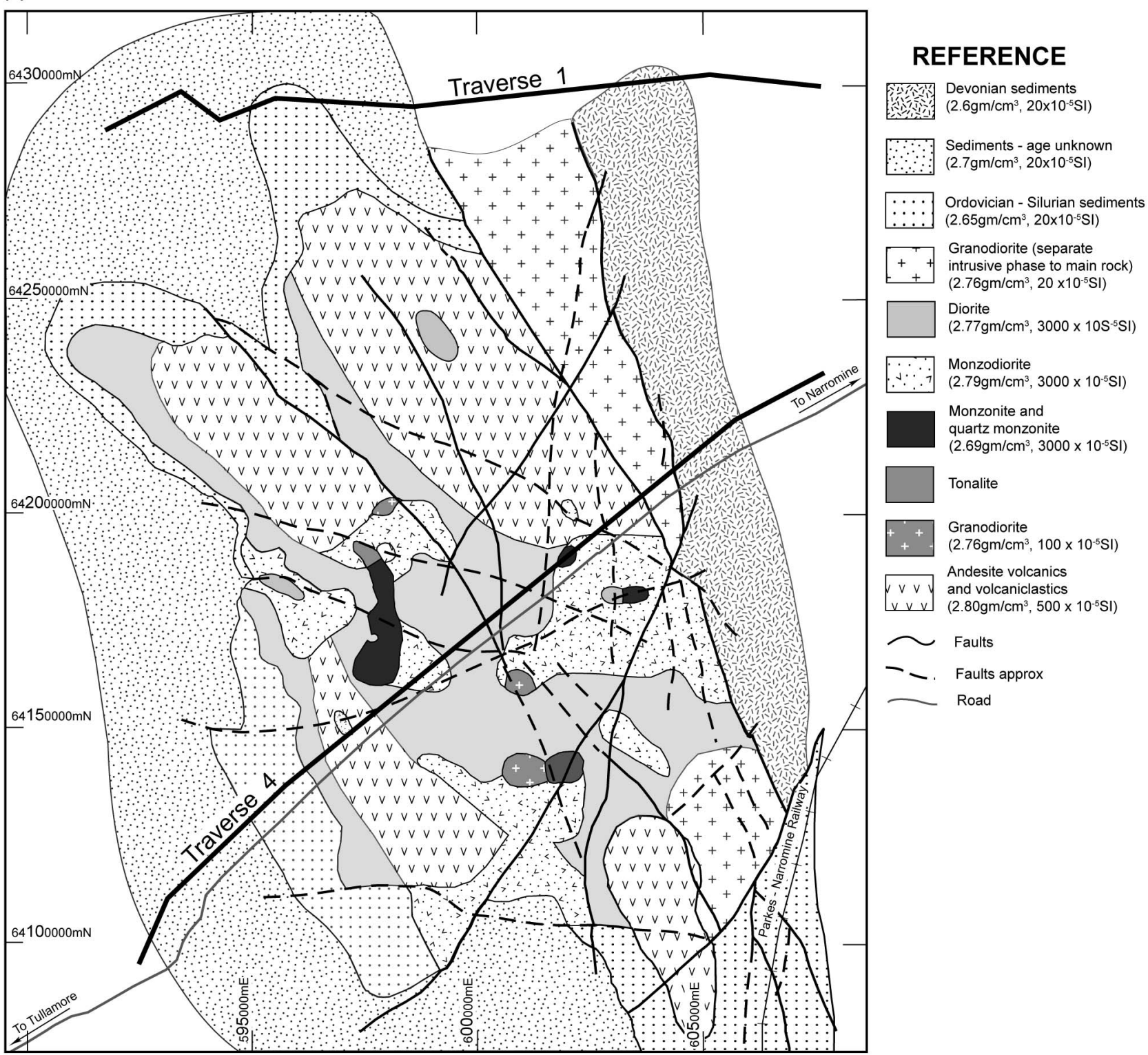

Grid Projection: MGA 94 Zone 55

Figure 3 Sketch geological map of the Narromine Igneous Complex (based on data from Goldfields Ltd and Resolute Ltd, and modified after Glen et al. 2007d) showing major intrusive units and interpreted extent of Ordovician volcanic and volcaniclastic rocks. Traverse lines 1 and 4 refer to a GS-NSW gravity survey discussed in Glen et al. (2007c).

are formerly glassy, sparsely plagioclase-phyric, trachytic-textured lavas. In drillhole NACD137, volcanic clasts and small, medium-grained holocrystalline diorite/ monzonitic clasts occur in a conglomerate, attesting to the unroofing and erosion of Early Ordovician(?) intrusive rocks regionally prior to intrusion of the main Narromine Igneous Complex intrusives. Volcanic/volcaniclastic rocks and andesitic/dioritic dykes show midgreenschist facies assemblages, with abundant epidote.

Andesitic lavas with good textural preservation are present in six drillholes (NACD24, NACD28, NACD31, NACD35, NACD38 and NACD49) and are mainly moderately plagioclase + augite-phyric. In a number of drillholes from the northern part of the complex, limited sample size and quality, and ambiguous textures in thin-section, made it impossible to determine whether some andesites are dykes emplaced at shallow crustal levels, or relatively thick lava flows.

Five samples of Narromine Igneous Complex lavas and dykes were analysed for major and trace elements (Table 1: see Appendix 1 for details of rock types analysed), two of these also for rare-earth elements (REE). Due to the small sample base, we have deliberately restricted this study to determining the affinities of these rocks, and comparing them with better-known suites described from the Parkes - Forbes - Cowal region (Crawford et al. 2007).

Apart from one shoshonitic andesite dyke in drillhole NACD105 that has petrographical and geochemical characteristics of the shoshonitic Phase 4 Late 
Table 1 Whole-rock compositions (recalculated to 100 volatilefree) of lavas and dykes from the Narromine Igneous Complex.

\begin{tabular}{|c|c|c|c|c|c|}
\hline Sample no. & $28-80.0$ & $96-96.0$ & $105-108.4$ & $137-110.0$ & $20-128.9$ \\
\hline Easting & 601113 & 601243 & 601033 & 598113 & 596113 \\
\hline Northing & 6422184 & 6412344 & 6413163 & 6416734 & 6420184 \\
\hline $\mathrm{SiO}_{2}$ & 63.36 & 67.93 & 65.24 & 47.72 & 53.31 \\
\hline $\mathrm{TiO}_{2}$ & 0.79 & 1.020 .82 & 0.4 & 0.86 & 0.82 \\
\hline $\mathrm{Al}_{2} \mathrm{O}_{3}$ & 16.09 & 17.42 & 17.37 & 20.23 & 18.26 \\
\hline $\mathrm{Fe}_{2} \mathrm{O}_{3}$ & 7.71 & 4.03 & 4.08 & 13.45 & 10.4 \\
\hline $\mathrm{MnO}$ & 0.12 & 0.04 & 0.11 & 0.79 & 0.28 \\
\hline $\mathrm{MgO}$ & 1.25 & 0.49 & 1.58 & 6.43 & 3.95 \\
\hline $\mathrm{CaO}$ & 1.45 & 1.04 & 3.5 & 4.18 & 5.9 \\
\hline $\mathrm{Na}_{2} \mathrm{O}$ & 4.71 & 4.51 & 5.17 & 5.08 & 3.73 \\
\hline $\mathrm{K}_{2} \mathrm{O}$ & 4.71 & 3.35 & 2.39 & 0.96 & 3.04 \\
\hline $\mathrm{P}_{2} \mathrm{O}_{5}$ & 0.35 & 0.37 & 0.15 & 0.28 & 0.32 \\
\hline LOI & 2.2 & 3.9 & 2.28 & 6.42 & 2.63 \\
\hline $\mathrm{Ni}$ & bdl & 3 & 7 & 16 & 9 \\
\hline $\mathrm{Cr}$ & 2 & 2 & 19 & 29 & 9 \\
\hline $\mathrm{V}$ & 48 & 65 & 80 & 271 & 277 \\
\hline $\mathrm{Sc}$ & 15 & 19 & 7 & 27 & 22 \\
\hline $\mathrm{Zr}$ & 329 & 202 & 120 & 56 & 83 \\
\hline $\mathrm{Nb}$ & 17.1 & 9.3 & 7 & 3 & 4.1 \\
\hline $\mathrm{Y}$ & 40.5 & 40.4 & 12 & 13 & 15.8 \\
\hline $\mathrm{Sr}$ & 213 & 402 & 855 & 575 & 1035 \\
\hline $\mathrm{Rb}$ & 57 & 51 & 32 & 19 & 35 \\
\hline $\mathrm{Ba}$ & 471 & 557 & 420 & 233 & 682 \\
\hline $\mathrm{Pb}$ & 5.3 & 4.8 & 5.0 & 6.5 & 3.1 \\
\hline $\mathrm{Zn}$ & 131 & 55 & 84 & 1627 & 164 \\
\hline $\mathrm{Cu}$ & 171 & 17 & 29 & 41 & 281 \\
\hline $\mathrm{La}$ & 34.8 & 25.1 & - & - & 14.4 \\
\hline $\mathrm{Ce}$ & 78.3 & 55.9 & - & - & 32.5 \\
\hline $\operatorname{Pr}$ & 10.68 & 7.91 & - & - & 4.54 \\
\hline $\mathrm{Nd}$ & 44.69 & 35.92 & - & - & 20.11 \\
\hline $\mathrm{Sm}$ & 10.06 & 8.77 & - & - & 4.91 \\
\hline $\mathrm{Eu}$ & 1.94 & 2.37 & - & - & 1.57 \\
\hline $\mathrm{Gd}$ & 8.54 & 8.96 & - & - & 4.2 \\
\hline $\mathrm{Tb}$ & 1.35 & 1.38 & - & - & 0.59 \\
\hline Dy & 7.72 & 7.65 & - & - & 3.18 \\
\hline Ho & 1.59 & 1.53 & - & - & 0.61 \\
\hline Er & 4.52 & 4.2 & - & - & 1.71 \\
\hline $\mathrm{Yb}$ & 4.36 & 3.73 & - & - & 1.53 \\
\hline $\mathrm{Lu}$ & 0.67 & 0.56 & - & - & 0.23 \\
\hline
\end{tabular}

bdl, below detection limit.

See Appendix 1 for rock types analysed.

Ordovician Goonumbla and Wombin Volcanics suite (including common apatite microphenocrysts) (Crawford et al. 2007), the remainder of the lavas and dykes from the Narromine Igneous Complex are more confidently correlated with the Phase 1 Early Ordovician Nelungaloo Volcanics and correlative lavas in the Cowal Igneous Complex further south (Figure 1). This is consistent with new SHRIMP dates of $c a 465 \mathrm{Ma}$ (Middle Ordovician) for monzodiorites intruding the volcanicvolcaniclastic package in the Narromine Igneous Complex (see below). A trachyandesite clast from drillhole NACD20, and two probable dykes from NACD28 and NACD96, show strong compositional affinities with the trachyte-trachyandesite suites from the main Cowal mine development at E42 (Golden Lava Unit) and from a drill prospect in the south-central part of the block at E43 (Glen et al. 2007b). This is true for both diagnostic trace-element ratios and REE patterns (Figure 4a).

\section{Intrusive rocks}

Two petrographically and geochemically distinct intrusive suites are present in the Narromine Igneous Complex. One suite, dominated by monzodiorite, but also including monzogabbros and monzonites, is far more abundant in drillcore. A second suite, composed mainly of rocks varying texturally from porphyritic dacite to holocrystalline granodiorite, occurs as sheetlike sills and dykes intruding the monzodiorite suite rocks, and also as a small pluton $(\sim 2 \times 1 \mathrm{~km})$ in the central part of the complex.

\section{MONZOGABBRO-MONZODIORITE-MONZONITE SUITE}

Abundant medium- to coarse-grained intrusive rocks in the Narromine Igneous Complex range from olivinebearing monzogabbros, through monzodiorites to subordinate monzonites (the latter present in drillholes NACD2, 77, 81, 85, 87 and 89). All these intrusive rocks show strong mineralogical and textural variation, even over several metres length of drillcore.

The monzogabbros contain occasional altered olivine in drillholes NACD90/136.8 $\mathrm{m}$ and $183.9 \mathrm{~m}$, and NACD131/71.8 $\mathrm{m}$, but are dominated by the assemblage Ca-plagioclase-augite-FeTioxides-apatite \pm hornblende \pm sphene, common interstitial primary brown biotite, and K-feldspar rimming plagioclase. Many show cumulate textures. Primary magmatic hornblende varies from abundant (drillholes NACD118/ $55.8 \mathrm{~m}$, NACD123/67.1 m, NACD122/65.8 m, NACD125/ $64.1 \mathrm{~m}$ ) to minor, and both hornblende and biotite are often replaced by chlorite and epidote, or actinolite in higher-grade rocks. Stout apatite crystals up to almost $1 \mathrm{~mm}$ long are common in the monzogabbros, and attest to the early crystallisation of apatite and the shoshonitic nature of the parental magmas to these rocks. With advancing fractionation through monzodioritic to monzonitic compositions, apatite generally decreases in abundance and occurs as more elongate, narrower crystals. Myrmekitic intergrowths are present as interstitial late crystallising material in some monzogabbros (e.g. drillhole NACD131/71.8 m), and interstitial primary quartz becomes an accessory phase in some monzodioritic rocks, although it is rare or absent in the monzonites.

\section{INTRUSIVE DACITE-GRANODIORITE SUITE}

A distinctive suite of shallow intrusive porphyritic dacite dykes intrudes the western part of the Narromine Igneous Complex. Intrusive dacites are well represented in drillholes NACD83 (115-150 m), and also occur in NACD82, 87, 89 and 91; in all instances, they intrude quartz-poor monzonitic to monzodioritic host rocks. A single, probably related but intensely silica-sericitepyrite-altered dacite (not analysed) was encountered in drillhole NACD98 in the eastern part of the intrusive complex, immediately against the eastern boundary fault of the Narromine Igneous Complex. The leastaltered dacite sample, from drillhole NACD83/116.6 m, is representative of the suite and is a plagioclase + quartz + hornblende + K-feldspar + FeTioxide + apatitephyric dacite with fresh hornblende and plagioclase 

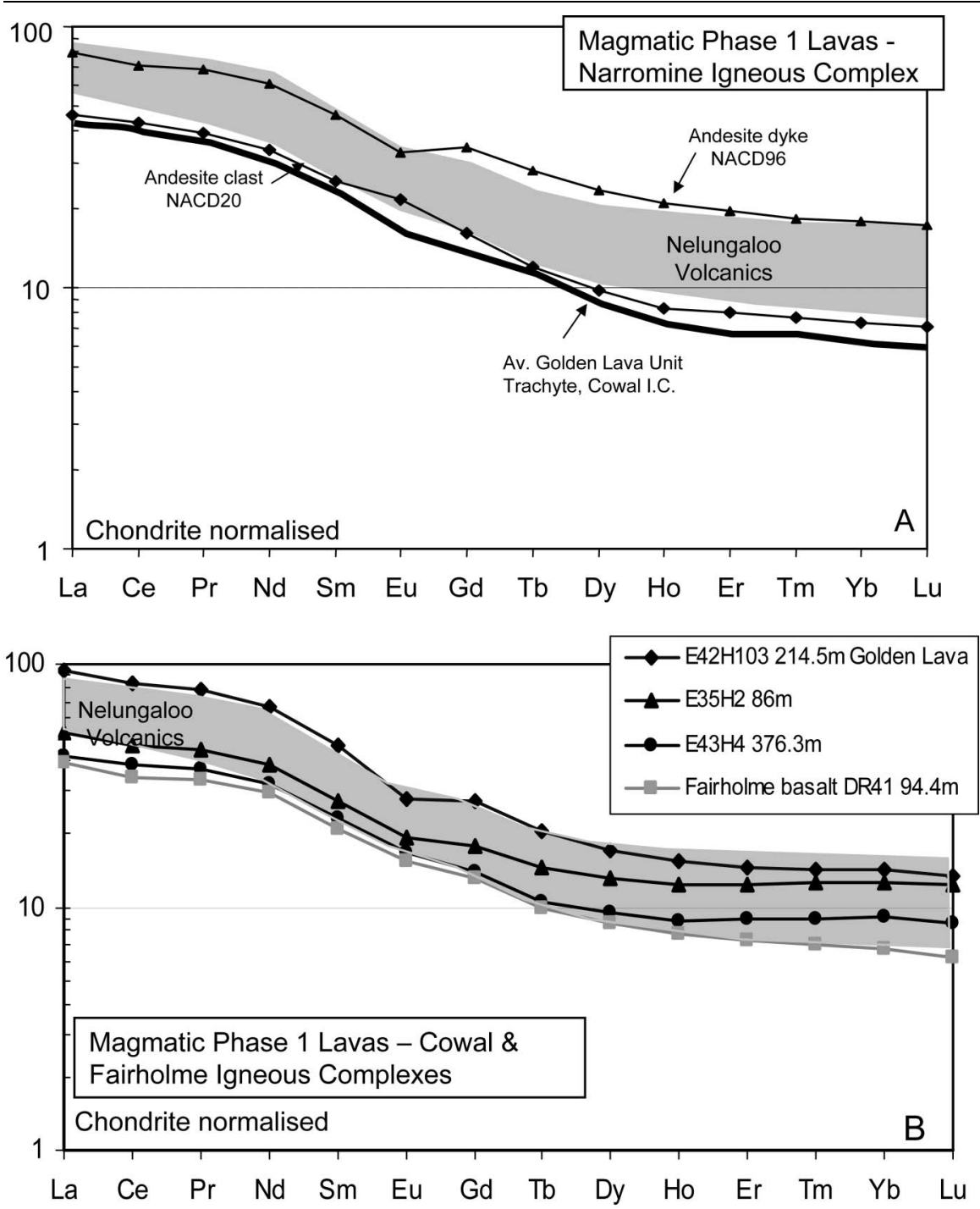

Figure 4 Chondrite-normalised REE patterns for Phase 1 lavas and dykes from the Junee-Narromine Volcanic Belt. (a) Narromine Igneous Complex. (b) Cowal Igneous Complex. In both panels, the field for lavas from the Early Ordovician Nelungaloo Volcanics (Glen et al. 2007b) is shown. Normalising values from Boynton (1984). phenocrysts, and rounded and resorbed quartz phenocrysts in a fine-grained quartzo-feldspathic groundmass. Hornblende phenocrysts are often replaced by chlorite.

Granodiorite occurs mainly in the central western part of Narromine Igneous Complex, as an interpreted $\sim 3.5 \times 1 \mathrm{~km}$ pluton (Figure 3). Granodioritic rocks compositionally identical to the dacite porphyries (see below) consist of blocky abitised plagioclase phenocrysts and occasional smaller hornblende and quartz phenocrysts, set in a groundmass of quartz, albite, K-feldspar and chlorite. The dacites are important, since they are associated with the limited chalcopyritepyrite mineralisation, and bear striking petrographic resemblance to host-rocks of the Copper Hill and Cargo mineralisation in the Molong Volcanic Belt. Tourmaline alteration has been noted in drillhole NACD11, associated with sericite-pyrite-carbonate alteration.

\section{Geochronology of Narromine Igneous Complex intrusive rocks}

The ages of five samples of Narromine Igneous Complex intrusive rocks have been determined using
SHRIMP $\mathrm{U}-\mathrm{Pb}$ dating of zircons: details of the dating methodology, using the AS3 and SL13 reference zircon standards, are given in Lickfold et al. (2007). Three dated samples are from the monzogabbromonzodiorite-monzonite (MMMz) suite, and two are from the intrusive dacite-granodiorite suite, including one dacite and one granodiorite. Of the former, a hornblende monzodiorite from drillhole NACD125 yielded an age of $461.4 \pm 2.6 \mathrm{Ma}$, a monzonite from drillhole NACD2 yielded an age of $463.3 \pm 3.7 \mathrm{Ma}$, and a monzodiorite from drillhole NACD137 yielded an age of $458.5 \pm 4.9 \mathrm{Ma}$ (Table 2; Figure $5 \mathrm{a}-\mathrm{c}$ ). These consistent ages indicate emplacement of the $\mathrm{MMMz}$ rocks broadly between 465 and $460 \mathrm{Ma}$, in midDarriwilian times at the end of the Middle Ordovician (following the time-scale in Percival \& Glen 2007 figure 2).

Two samples of the intrusive dacite-granodiorite suite from the Narromine Igneous Complex were also dated: a dacite from drillhole NACD83 and a granodiorite from NACD 86. These show almost identical complex age spectra for the analysed grains (Figure $5 \mathrm{~d}$, e). Numerous grains have obvious rounded cores interpreted to be inherited zircons. These cores yielded dates 
of $467.3 \pm 4.8 \mathrm{Ma}$ in the dacite and $470.6 \pm 5.5 \mathrm{Ma}$ in the granodiorite, only very slightly older than (and within error of) the dates determined for the Narromine Igneous Complex MMMz suite rocks. However, all zircons studied in both the dacite and the granodiorite have well-developed euhedral outer zones that record ages of $440.9 \pm 4.5 \mathrm{Ma}$ in the dacite and $449.4 \pm 3.9 \mathrm{Ma}$ in the granodiorite (Table 2; Figure $5 \mathrm{~d}$, e). Averaging at $445 \mathrm{Ma}$ (mid-Bolindian, Late Ordovician), these dates agree well within error with available dates for petrographically and compositionally similar intrusive dacites from both Copper Hill and Cargo in the Molong Belt (453-450 Ma) (Crawford et al. 2007). A widespread but relatively small volume, medium-K calc-alkaline intrusive event is therefore well documented in the Ordovician belts of central western New South Wales at ca 455-445 Ma. We refer to this suite of intrusive dacites and granodiorites as the Copper Hill Suite, after the Copper Hill area near Molong, where rocks of this suite were first thoroughly described (Blevin 2002).

We have no age constraints on the volcanic and volcaniclastic host-rocks to these Darriwilian intrusives in the Narromine Igneous Complex. This 465$460 \mathrm{Ma}$ intrusive event is also well represented in the Cowal Igneous Complex (see below), but the single pre-Late Ordovician intrusive complex dated in the Forbes-Parkes region, the Nelungaloo Intrusives, is significantly older at $481 \pm 3 \mathrm{Ma}$ (Butera et al. 2001; Simpson et al. 2005). In the Molong Belt, Darriwilian rocks comprise the Fairbridge Volcanics, the Cargo Volcanics and possibly the lower Blayney Volcanics, but no contemporaneous holocrystalline intrusive complexes are known from this belt.

\section{Geochemistry of Narromine Igneous Complex intrusive rocks}

Representative whole-rock major- and trace-element analyses of 29 least-altered intrusive rocks (excluding narrow dykes) from the Narromine Igneous Complex (seven from the Copper Hill Suite) are given in Table 3 (see Appendix 1 for details of rock types analysed): analytical methods are given in Squire and Crawford (2007). Samples were chosen to cover the geographic and petrographic-compositional range represented among the 125 rocks examined in thin-section.

\section{HOLOCRYSTALLINE MONZOGABBRO - MONZODIORITE - MONZONITE SUITE}

The Narromine Igneous Complex holocrystalline intrusive rocks of the $\mathrm{MMMz}$ suite define coherent fractionation trends (Figure 6) from relatively low-Mg monzogabbroic rocks to monzodioritic and monzonitic compositions with $\sim 54-62 \% \mathrm{SiO}_{2}$; a single quartz monzonite has $66 \% \mathrm{SiO}_{2}$. The monzogabbros have compositions that trend to lower $\mathrm{SiO}_{2}, \mathrm{Fe}_{2} \mathrm{O}_{3}$ and $\mathrm{TiO}_{2}$ (Figure 6) than other rocks in the suite that lack cumulate textures, reflecting accumulation of plagioclase + clinopyroxene + hornblende \pm apatite. High $\mathrm{P}_{2} \mathrm{O}_{5}$ contents in monzogabbros (Table 3) indicate early apatite fractionation, a characteristic of high-K calc-alkaline and shoshonitic lavas. The MMMz suite rocks show mainly high-K calc-alkaline affinities (Figure 6), definitively so for the least-altered rocks; the two monzogabbroic rocks that plot in the low-K field are the most plagioclase + clinopyroxenerich, hornblende-poor cumulates and are not liquid compositions.

REE patterns for three samples from across the fractionation range of the MMMz suite are shown in Figure $7 \mathrm{a}$. The pattern for a cumulate-textured monzogabbro from drillhole NACD82 reflects the accumulation of plagioclase, clinopyroxene, amphibole and apatite (note positive $\mathrm{Eu}$ anomaly), whereas the other two patterns are for rocks that are petrographically more appropriate for near-liquid compositions. Fractionation from monzogabbro with $54 \% \mathrm{SiO}_{2}$ and $3.9 \% \mathrm{MgO}$ to one with $60 \% \mathrm{SiO}_{2}$ and $2.3 \% \mathrm{MgO}$ produced a decrease in total REE abundances (Figure 7a), with a steeper drop in MREE than HREE, a feature usually attributable to plagioclase-hornblende-apatite fractionation, and in keeping with the observed phenocryst assemblages. N-MORB normalised multi-element patterns are typical of high-K calc-alkaline and shoshonitic lavas in modern western Pacific arc systems (Figure 7c), and are close to those for plagioclase-phyric lavas from the Walli Volcanics in the Molong Volcanic Belt that also show affinities extending from high-K calc-alkaline to shoshonitic (Crawford et al. 2007; Simpson et al. 2007). A monzogabbro from NACD97 has an initial $\varepsilon_{\mathrm{Nd}}$ value of +7.4 calculated at $465 \mathrm{Ma}$, within the range for the Phase 2 Cargo Volcanics of the same age in the Molong Volcanic Belt (Crawford et al. 2007).

\section{COPPER HILL SUITE INTRUSIVE DACITES AND GRANODIORITES}

Despite strikingly different textures and grainsize, the porphyritic dacite dykes are compositionally identical to the granodioritic rocks in the western part of the Narromine Igneous Complex, with a relatively restricted $\mathrm{SiO}_{2}$ range, and together they define coherent and restricted compositional fields on plots against $\mathrm{SiO}_{2}$ (Figure 6). The dacite-granodiorite compositional fields for many elements and element ratios are clearly separate from those for the $\mathrm{MMMz}$ trends. This is especially evident for the $\mathrm{K}_{2} \mathrm{O}, \mathrm{TiO}_{2}, \mathrm{Zr}, \mathrm{Y}, \mathrm{Nb}$ and $\mathrm{Cr}$ vs $\mathrm{SiO}_{2}$ plots $\left(\mathrm{K}_{2} \mathrm{O}, \mathrm{TiO}_{2}, \mathrm{Cr}\right.$ and $\mathrm{Zr}$ shown in Figure 6), where the more evolved rocks have consistently lower $\mathrm{K}_{2} \mathrm{O}, \mathrm{Zr}$ and $\mathrm{Nb}$ contents, and notably higher $\mathrm{Cr}$ contents than the monzodiorites. No reasonable fractionation scheme can drive the MMMz monzodiorites towards the compositional fields defined by the Copper Hill Suite dacite-granodiorite rocks. Note that a single member of the $\mathrm{MMMz}$ suite has $66.7 \% \mathrm{SiO}_{2}$, like the less-evolved dacites, but that this sample has $\mathrm{Zr}, \mathrm{Y}$ and $\mathrm{Nb}$ contents more than twice as high as the intrusive dacite-granodiorite suite rocks at the same $\mathrm{SiO}_{2}$ level. As indicated by $\mathrm{U}-\mathrm{Pb}$ SHRIMP zircon dating, the medium-K calc-alkaline Copper Hill Suite dacite-granodiorite intrusions post-date the high-K calc-alkaline to shoshonitic $\mathrm{MMMz}$ suite by $15-20$ million years. 
Table 2 SHRIMP U-Pb zircon results for samples from the Narromine Igneous Complex.

\begin{tabular}{|c|c|c|c|c|c|c|c|c|c|c|c|c|c|c|}
\hline \multirow{2}{*}{$\begin{array}{l}\text { Grain } \\
\text { spot }\end{array}$} & \multirow{2}{*}{$\begin{array}{c}\mathrm{U} \\
(\mathrm{ppm})\end{array}$} & \multirow{2}{*}{$\begin{array}{c}\text { Th } \\
\text { (ppm) }\end{array}$} & \multirow[t]{2}{*}{$\mathrm{Th} / \mathrm{U}$} & \multirow{2}{*}{$\begin{array}{l}{ }^{206} \mathrm{~Pb}^{*} \\
(\mathrm{ppm})\end{array}$} & \multirow[t]{2}{*}{${ }^{204} \mathrm{~Pb} /{ }^{206} \mathrm{~Pb}$} & \multirow{2}{*}{$\begin{array}{l}f_{206} \\
(\%)\end{array}$} & \multicolumn{8}{|c|}{ Total radiogenic age (Ma) } \\
\hline & & & & & & & ${ }^{238} \mathrm{U} /{ }^{206} \mathrm{~Pb}$ & \pm & $\begin{array}{c}{ }^{207} \mathrm{~Pb} / \\
{ }^{206} \mathrm{~Pb}\end{array}$ & \pm & $\begin{array}{c}{ }^{206} \mathrm{~Pb} / \\
{ }^{238} \mathrm{U}\end{array}$ & \pm & $\begin{array}{c}{ }^{206} \mathrm{~Pb} / \\
{ }^{238} \mathrm{U}\end{array}$ & \pm \\
\hline \multicolumn{15}{|c|}{ NACD 137, 119-120 m (598113E, 6416734N) Age $458.5 \pm 5.9 \mathrm{Ma}$} \\
\hline 1.1 & 132 & 66 & 0.50 & 8.5 & 0.000249 & 0.29 & 13.380 & 0.221 & 0.0586 & 0.0015 & 0.0745 & 0.0013 & 463.3 & 7.5 \\
\hline 2.1 & 60 & 28 & 0.47 & 3.7 & - & 0.78 & 14.010 & 0.252 & 0.0621 & 0.0022 & 0.0708 & 0.0013 & 441.1 & 7.8 \\
\hline 3.1 & 149 & 82 & 0.55 & 9.3 & 0.000591 & 0.46 & 13.763 & 0.190 & 0.0597 & 0.0015 & 0.0723 & 0.0010 & 450.1 & 6.2 \\
\hline 4.1 & 112 & 52 & 0.47 & 7.1 & 0.000285 & 0.02 & 13.652 & 0.271 & 0.0563 & 0.0018 & 0.0732 & 0.0015 & 455.6 & 8.9 \\
\hline 5.1 & 85 & 30 & 0.36 & 5.5 & - & 0.16 & 13.271 & 0.217 & 0.0577 & 0.0017 & 0.0752 & 0.0013 & 467.6 & 7.6 \\
\hline 6.1 & 97 & 52 & 0.53 & 6.2 & - & 0.12 & 13.380 & 0.217 & 0.0573 & 0.0017 & 0.0746 & 0.0012 & 464.1 & 7.4 \\
\hline 7.1 & 127 & 47 & 0.37 & 8.0 & - & 0.16 & 13.618 & 0.199 & 0.0574 & 0.0014 & 0.0733 & 0.0011 & 456.1 & 6.6 \\
\hline 8.1 & 165 & 83 & 0.50 & 10.5 & 0.000629 & 0.12 & 13.481 & 0.186 & 0.0572 & 0.0012 & 0.0741 & 0.0010 & 460.7 & 6.3 \\
\hline 9.1 & 154 & 53 & 0.34 & 9.5 & - & 0.45 & 13.917 & 0.189 & 0.0594 & 0.0014 & 0.0715 & 0.0010 & 445.4 & 6.0 \\
\hline 10.1 & 146 & 57 & 0.39 & 9.1 & 0.000806 & -0.04 & 13.768 & 0.191 & 0.0557 & 0.0014 & 0.0727 & 0.0010 & 452.2 & 6.2 \\
\hline 11.1 & 149 & 48 & 0.32 & 9.8 & - & -0.22 & 13.164 & 0.179 & 0.0547 & 0.0012 & 0.0761 & 0.0011 & 473.0 & 6.3 \\
\hline 12.1 & 70 & 35 & 0.50 & 4.6 & 0.000119 & -0.16 & 12.938 & 0.211 & 0.0554 & 0.0019 & 0.0774 & 0.0013 & 480.7 & 7.7 \\
\hline 13.1 & 46 & 16 & 0.36 & 2.9 & 0.000325 & 0.82 & 13.373 & 0.341 & 0.0629 & 0.0025 & 0.0742 & 0.0019 & 461.2 & 11.6 \\
\hline 14.1 & 48 & 17 & 0.35 & 3.0 & 0.000207 & 1.52 & 13.940 & 0.263 & 0.0680 & 0.0026 & 0.0706 & 0.0014 & 440.0 & 8.3 \\
\hline 15.1 & 216 & 86 & 0.40 & 12.3 & 0.001045 & 1.53 & 15.076 & 0.196 & 0.0672 & 0.0014 & 0.0653 & 0.0009 & 407.9 & 5.3 \\
\hline 16.1 & 106 & 44 & 0.42 & 6.8 & - & 1.26 & 13.366 & 0.204 & 0.0664 & 0.0021 & 0.0739 & 0.0012 & 459.5 & 7.0 \\
\hline 17.1 & 69 & 33 & 0.48 & 4.5 & 0.001495 & -0.35 & 13.162 & 0.236 & 0.0537 & 0.0019 & 0.0762 & 0.0014 & 473.6 & 8.4 \\
\hline 18.1 & 55 & 16 & 0.30 & 3.4 & - & 0.42 & 13.915 & 0.252 & 0.0592 & 0.0029 & 0.0716 & 0.0013 & 445.6 & 8.1 \\
\hline 19.1 & 41 & 14 & 0.34 & 2.7 & - & 0.06 & 12.756 & 0.249 & 0.0574 & 0.0024 & 0.0783 & 0.0016 & 486.2 & 9.4 \\
\hline 20.1 & 43 & 13 & 0.30 & 2.8 & 0.000628 & 1.13 & 13.382 & 0.254 & 0.0654 & 0.0026 & 0.0739 & 0.0014 & 459.5 & 8.7 \\
\hline
\end{tabular}

NACD 125, 64.1-64.4 m (604613E, 6417684N) Age 461.4 $\pm 4.2 \mathrm{Ma}$

\begin{tabular}{|c|c|c|c|c|c|c|c|c|c|c|c|c|c|}
\hline 1.1 & 1191 & 907 & 0.76 & 77.1 & - & $<0.01$ & 13.270 & 0.157 & 0.0561 & 0.0004 & 0.0754 & 0.0009 & 468.5 \\
\hline 2.1 & 212 & 91 & 0.43 & 13.7 & 0.000043 & $<0.01$ & 13.312 & 0.167 & 0.0552 & 0.0010 & 0.0752 & 0.0010 & 467.6 \\
\hline 3.1 & 339 & 144 & 0.43 & 21.5 & 0.000104 & 0.24 & 13.560 & 0.191 & 0.0581 & 0.0008 & 0.0736 & 0.0011 & 457.6 \\
\hline 4.1 & 403 & 195 & 0.48 & 26.7 & 0.000070 & $<0.01$ & 12.946 & 0.149 & 0.0558 & 0.0007 & 0.0773 & 0.0009 & 480.1 \\
\hline 5.1 & 407 & 217 & 0.53 & 25.9 & - & $<0.01$ & 13.482 & 0.157 & 0.0560 & 0.0007 & 0.0742 & 0.0009 & 461.3 \\
\hline 6.1 & 173 & 71 & 0.41 & 11.4 & 0.000286 & 0.10 & 13.019 & 0.169 & 0.0574 & 0.0011 & 0.0767 & 0.0010 & 476.6 \\
\hline 7.1 & 155 & 57 & 0.37 & 9.9 & 0.000703 & 0.35 & 13.450 & 0.178 & 0.0591 & 0.0012 & 0.0741 & 0.0010 & 460.7 \\
\hline 8.1 & 175 & 57 & 0.33 & 11.0 & 0.000443 & 0.46 & 13.607 & 0.173 & 0.0598 & 0.0011 & 0.0732 & 0.0009 & 455.1 \\
\hline 9.1 & 286 & 122 & 0.43 & 18.5 & - & $<0.01$ & 13.243 & 0.229 & 0.0543 & 0.0008 & 0.0757 & 0.0013 & 470.5 \\
\hline 10.1 & 969 & 936 & 0.97 & 60.7 & 0.000025 & 0.04 & 13.727 & 0.148 & 0.0564 & 0.0004 & 0.0728 & 0.0008 & 453.1 \\
\hline 11.1 & 298 & 147 & 0.49 & 19.1 & - & 0.01 & 13.428 & 0.157 & 0.0564 & 0.0008 & 0.0745 & 0.0009 & 463.0 \\
\hline 12.1 & 945 & 690 & 0.73 & 59.5 & 0.000065 & $<0.01$ & 13.646 & 0.163 & 0.0561 & 0.0006 & 0.0733 & 0.0009 & 455.9 \\
\hline 13.1 & 307 & 134 & 0.44 & 19.9 & 0.000030 & 0.27 & 13.287 & 0.156 & 0.0585 & 0.0008 & 0.0751 & 0.0009 & 466.6 \\
\hline 14.1 & 490 & 269 & 0.55 & 30.2 & - & 0.12 & 13.943 & 0.157 & 0.0568 & 0.0006 & 0.0716 & 0.0008 & 446.0 \\
\hline 15.1 & 843 & 512 & 0.61 & 53.1 & 0.000041 & $<0.01$ & 13.646 & 0.147 & 0.0558 & 0.0005 & 0.0733 & 0.0008 & 456.1 \\
\hline 16.1 & 202 & 83 & 0.41 & 12.9 & 0.000026 & 0.07 & 13.478 & 0.166 & 0.0568 & 0.0010 & 0.0741 & 0.0009 & 461.0 \\
\hline 17.1 & 503 & 237 & 0.47 & 32.8 & - & 0.02 & 13.165 & 0.149 & 0.0567 & 0.0006 & 0.0759 & 0.0009 & 471.9 \\
\hline 18.1 & 443 & 230 & 0.52 & 28.3 & 0.000067 & 0.10 & 13.478 & 0.154 & 0.0571 & 0.0007 & 0.0741 & 0.0009 & 460.9 \\
\hline 19.1 & 474 & 229 & 0.48 & 30.0 & - & 0.08 & 13.581 & 0.166 & 0.0568 & 0.0006 & 0.0736 & 0.0009 & 457.7 \\
\hline 20.1 & 85 & 26 & 0.31 & 5.4 & - & 0.22 & 13.386 & 0.196 & 0.0581 & 0.0015 & 0.0745 & 0.0011 & 463.4 \\
\hline
\end{tabular}

NACD 2, 82-83 m (603113E, 6418184N) Age 463.3 \pm 5.0 Ma

\begin{tabular}{|c|c|c|c|c|c|c|c|c|c|c|c|c|c|c|}
\hline 1.1 & 266 & 122 & 0.46 & 16.6 & 0.000667 & 0.10 & 13.771 & 0.174 & 0.0568 & 0.0010 & 0.0725 & 0.0009 & 451.4 & 5.6 \\
\hline 2.1 & 115 & 44 & 0.38 & 7.3 & 0.000439 & 0.42 & 13.553 & 0.201 & 0.0595 & 0.0015 & 0.0735 & 0.0011 & 457.1 & 6.7 \\
\hline 3.1 & 268 & 127 & 0.47 & 17.6 & - & 0.03 & 13.089 & 0.218 & 0.0568 & 0.0010 & 0.0764 & 0.0013 & 474.5 & 7.8 \\
\hline 4.1 & 335 & 87 & 0.26 & 21.5 & 0.000082 & 0.10 & 13.379 & 0.163 & 0.0571 & 0.0008 & 0.0747 & 0.0009 & 464.2 & 5.5 \\
\hline 5.1 & 137 & 39 & 0.29 & 8.9 & 0.000080 & 0.32 & 13.149 & 0.185 & 0.0591 & 0.0013 & 0.0758 & 0.0011 & 471.1 & 6.5 \\
\hline 6.1 & 181 & 48 & 0.27 & 11.8 & - & 0.01 & 13.126 & 0.177 & 0.0566 & 0.0012 & 0.0762 & 0.0010 & 473.3 & 6.3 \\
\hline 7.1 & 410 & 195 & 0.48 & 24.9 & - & 0.08 & 14.122 & 0.174 & 0.0563 & 0.0008 & 0.0708 & 0.0009 & 440.7 & 5.3 \\
\hline 8.1 & 389 & 171 & 0.44 & 22.4 & 0.000228 & 0.17 & 14.928 & 0.180 & 0.0565 & 0.0008 & 0.0669 & 0.0008 & 417.3 & 5.0 \\
\hline 9.1 & 380 & 199 & 0.52 & 23.3 & - & 0.31 & 13.983 & 0.166 & 0.0583 & 0.0008 & 0.0713 & 0.0009 & 443.9 & 5.2 \\
\hline 10.1 & 222 & 94 & 0.42 & 14.0 & 0.000083 & 0.17 & 13.632 & 0.162 & 0.0574 & 0.0007 & 0.0732 & 0.0009 & 455.6 & 5.3 \\
\hline 11.1 & 414 & 182 & 0.44 & 26.5 & 0.000120 & 0.19 & 13.387 & 0.150 & 0.0578 & 0.0005 & 0.0746 & 0.0009 & 463.6 & 5.1 \\
\hline 12.1 & 330 & 152 & 0.46 & 20.6 & 0.000010 & 0.09 & 13.773 & 0.155 & 0.0567 & 0.0006 & 0.0725 & 0.0008 & 451.4 & 5.0 \\
\hline 13.1 & 229 & 66 & 0.29 & 14.3 & 0.000198 & 0.20 & 13.740 & 0.162 & 0.0576 & 0.0012 & 0.0726 & 0.0009 & 452.0 & 5.3 \\
\hline 14.1 & 452 & 196 & 0.43 & 29.0 & 0.000022 & 0.02 & 13.399 & 0.147 & 0.0564 & 0.0005 & 0.0746 & 0.0008 & 463.9 & 5.0 \\
\hline 15.1 & 375 & 153 & 0.41 & 24.1 & 0.000226 & $<0.01$ & 13.370 & 0.149 & 0.0562 & 0.0006 & 0.0748 & 0.0008 & 465.0 & 5.1 \\
\hline 16.1 & 402 & 189 & 0.47 & 26.1 & 0.000058 & 0.08 & 13.242 & 0.147 & 0.0571 & 0.0006 & 0.0755 & 0.0009 & 468.9 & 5.1 \\
\hline 17.1 & 434 & 198 & 0.46 & 27.8 & 0.000070 & 0.01 & 13.415 & 0.168 & 0.0564 & 0.0005 & 0.0745 & 0.0010 & 463.4 & 5.7 \\
\hline
\end{tabular}


Table 2 (Continued).

\begin{tabular}{|c|c|c|c|c|c|c|c|c|c|c|c|c|c|c|}
\hline \multirow{2}{*}{$\begin{array}{l}\text { Grain } \\
\text { spot }\end{array}$} & \multirow{2}{*}{$\begin{array}{c}\mathrm{U} \\
(\mathrm{ppm})\end{array}$} & \multirow{2}{*}{$\begin{array}{l}\text { Th } \\
\text { (ppm) }\end{array}$} & \multirow[t]{2}{*}{$\mathrm{Th} / \mathrm{U}$} & \multirow{2}{*}{$\begin{array}{l}{ }^{206} \mathrm{~Pb}^{*} \\
(\mathrm{ppm})\end{array}$} & \multirow[t]{2}{*}{${ }^{204} \mathrm{~Pb} /{ }^{206} \mathrm{~Pb}$} & \multirow{2}{*}{$\begin{array}{l}f_{206} \\
(\%)\end{array}$} & \multicolumn{8}{|c|}{ Total radiogenic age $(\mathrm{Ma})$} \\
\hline & & & & & & & ${ }^{238} \mathrm{U} /{ }^{206} \mathrm{~Pb}$ & \pm & $\begin{array}{l}{ }^{207} \mathrm{~Pb} / \\
{ }^{206} \mathrm{~Pb}\end{array}$ & \pm & $\begin{array}{c}{ }^{206} \mathrm{~Pb} / \\
{ }^{238} \mathrm{U}\end{array}$ & \pm & $\begin{array}{c}{ }^{206} \mathrm{~Pb} / \\
{ }^{238} \mathrm{U}\end{array}$ & \pm \\
\hline 18.1 & 312 & 126 & 0.40 & 20.3 & 0.000228 & $<0.01$ & 13.208 & 0.151 & 0.0561 & 0.0006 & 0.0757 & 0.0009 & 470.7 & 5.3 \\
\hline 19.1 & 291 & 134 & 0.46 & 19.0 & 0.000000 & 0.04 & 13.141 & 0.148 & 0.0568 & 0.0006 & 0.0761 & 0.0009 & 472.6 & 5.2 \\
\hline 4.2 & 356 & 166 & 0.47 & 22.8 & 0.000089 & $<0.01$ & 13.408 & 0.148 & 0.0562 & 0.0006 & 0.0746 & 0.0008 & 463.7 & 5.0 \\
\hline 5.2 & 191 & 46 & 0.24 & 12.3 & 0.000277 & 0.16 & 13.303 & 0.161 & 0.0577 & 0.0008 & 0.0750 & 0.0009 & 466.5 & 5.5 \\
\hline
\end{tabular}

NACD 86, 129-130 m (598004E, 6418437N) Age: younger group 449.4 \pm 5.0 Ma; older group 470.6 \pm 5.5 Ma

\begin{tabular}{|c|c|c|c|c|c|c|c|c|c|c|c|c|c|}
\hline 1.1 & 147 & 91 & 0.62 & 9.1 & 0.000092 & 0.32 & 13.850 & 0.202 & 0.0585 & 0.0014 & 0.0720 & 0.0011 & 448.0 \\
\hline 2.1 & 43 & 15 & 0.36 & 2.6 & 0.001923 & 0.03 & 14.419 & 0.323 & 0.0557 & 0.0029 & 0.0693 & 0.0016 & 432.1 \\
\hline 2.2 & 71 & 29 & 0.40 & 4.8 & - & 0.48 & 12.736 & 0.232 & 0.0607 & 0.0024 & 0.0781 & 0.0015 & 485.0 \\
\hline 3.1 & 251 & 183 & 0.73 & 15.4 & - & 0.29 & 14.005 & 0.184 & 0.0581 & 0.0010 & 0.0712 & 0.0010 & 443.4 \\
\hline 4.1 & 48 & 19 & 0.39 & 3.1 & - & 0.15 & 13.238 & 0.270 & 0.0576 & 0.0025 & 0.0754 & 0.0016 & 468.8 \\
\hline 5.1 & 92 & 43 & 0.47 & 5.5 & 0.000160 & 0.23 & 14.269 & 0.229 & 0.0574 & 0.0017 & 0.0699 & 0.0011 & 435.7 \\
\hline 6.1 & 165 & 119 & 0.72 & 10.1 & - & 0.25 & 14.133 & 0.200 & 0.0577 & 0.0014 & 0.0706 & 0.0010 & 439.7 \\
\hline 6.2 & 90 & 49 & 0.55 & 5.9 & 0.000536 & $<0.01$ & 13.151 & 0.218 & 0.0557 & 0.0027 & 0.0761 & 0.0013 & 472.9 \\
\hline 7.1 & 156 & 118 & 0.76 & 9.3 & - & 0.17 & 14.391 & 0.214 & 0.0569 & 0.0014 & 0.0694 & 0.0011 & 432.3 \\
\hline 8.1 & 50 & 16 & 0.31 & 3.1 & 0.001303 & 1.00 & 14.107 & 0.277 & 0.0637 & 0.0023 & 0.0702 & 0.0014 & 437.2 \\
\hline 9.1 & 102 & 46 & 0.45 & 6.6 & - & 0.21 & 13.360 & 0.206 & 0.0580 & 0.0016 & 0.0747 & 0.0012 & 464.4 \\
\hline 10.1 & 149 & 89 & 0.60 & 9.5 & - & 0.16 & 13.379 & 0.196 & 0.0576 & 0.0014 & 0.0746 & 0.0011 & 464.0 \\
\hline 10.2 & 105 & 48 & 0.46 & 6.7 & - & 0.70 & 13.458 & 0.184 & 0.0618 & 0.0012 & 0.0738 & 0.0010 & 458.9 \\
\hline 11.1 & 119 & 72 & 0.61 & 7.7 & 0.000224 & 0.22 & 13.198 & 0.168 & 0.0582 & 0.0010 & 0.0756 & 0.0010 & 469.9 \\
\hline 12.1 & 175 & 69 & 0.39 & 11.2 & 0.000352 & 0.28 & 13.433 & 0.161 & 0.0585 & 0.0009 & 0.0742 & 0.0009 & 461.6 \\
\hline 13.1 & 89 & 33 & 0.37 & 5.7 & 0.000106 & 0.49 & 13.375 & 0.223 & 0.0602 & 0.0012 & 0.0744 & 0.0013 & 462.6 \\
\hline 14.1 & 87 & 43 & 0.50 & 5.7 & - & 0.47 & 13.122 & 0.179 & 0.0603 & 0.0012 & 0.0759 & 0.0011 & 471.3 \\
\hline 15.1 & 88 & 30 & 0.34 & 5.8 & 0.000516 & 0.58 & 13.103 & 0.178 & 0.0612 & 0.0012 & 0.0759 & 0.0011 & 471.5 \\
\hline 16.1 & 183 & 99 & 0.54 & 11.7 & 0.000321 & 0.19 & 13.451 & 0.185 & 0.0577 & 0.0008 & 0.0742 & 0.0010 & 461.5 \\
\hline 17.1 & 75 & 23 & 0.30 & 4.7 & 0.000435 & 1.00 & 13.792 & 0.198 & 0.0640 & 0.0014 & 0.0718 & 0.0010 & 446.9 \\
\hline 18.1 & 93 & 41 & 0.44 & 6.1 & 0.000571 & 0.47 & 13.053 & 0.182 & 0.0604 & 0.0012 & 0.0762 & 0.0011 & 473.7 \\
\hline 19.1 & 45 & 13 & 0.29 & 3.0 & 0.000887 & 1.32 & 13.103 & 0.213 & 0.0671 & 0.0017 & 0.0753 & 0.0013 & 468.0 \\
\hline 0.1 & 47 & 14 & 0.29 & 3.0 & 0.000923 & 1.16 & 13.704 & 0.272 & 0.0653 & 0.0017 & 0.0721 & 0.0015 & 448.9 \\
\hline
\end{tabular}

NACD 83, $116 \mathrm{~m}(598114 \mathrm{E}, 6417687 \mathrm{~N})$ Age: younger group 440.9 $\pm 5.5 \mathrm{Ma}$; older group $467.3 \pm 4.8 \mathrm{Ma}$

\begin{tabular}{|c|c|c|c|c|c|c|c|c|c|c|c|c|c|}
\hline 1.1 & 75 & 34 & 0.45 & 4.7 & 0.000057 & 0.18 & 13.758 & 0.269 & 0.0574 & 0.0023 & 0.0726 & 0.0015 & 451.5 \\
\hline 2.1 & 71 & 24 & 0.34 & 4.2 & 0.000373 & 0.19 & 14.562 & 0.260 & 0.0569 & 0.0022 & 0.0685 & 0.0013 & 427.4 \\
\hline 3.1 & 91 & 28 & 0.31 & 5.3 & 0.002959 & 1.35 & 14.677 & 0.234 & 0.0661 & 0.0019 & 0.0672 & 0.0011 & 419.3 \\
\hline 4.1 & 73 & 28 & 0.38 & 4.7 & 0.000350 & 0.57 & 13.319 & 0.241 & 0.0609 & 0.0019 & 0.0747 & 0.0014 & 464.1 \\
\hline 5.1 & 204 & 162 & 0.79 & 12.9 & 0.000263 & 0.40 & 13.606 & 0.177 & 0.0593 & 0.0011 & 0.0732 & 0.0010 & 455.4 \\
\hline 6.1 & 237 & 314 & 1.32 & 16.7 & 0.000202 & $<0.01$ & 12.186 & 0.158 & 0.0566 & 0.0010 & 0.0821 & 0.0011 & 508.9 \\
\hline 7.1 & 100 & 40 & 0.40 & 6.2 & 0.001186 & 0.39 & 13.769 & 0.217 & 0.0591 & 0.0016 & 0.0723 & 0.0012 & 450.3 \\
\hline 8.1 & 538 & 511 & 0.95 & 33.3 & - & $<0.01$ & 13.888 & 0.162 & 0.0552 & 0.0007 & 0.0721 & 0.0009 & 448.6 \\
\hline 9.1 & 63 & 24 & 0.39 & 3.9 & - & 0.39 & 13.809 & 0.249 & 0.0591 & 0.0021 & 0.0721 & 0.0013 & 449.0 \\
\hline 10.1 & 56 & 18 & 0.32 & 3.6 & 0.000602 & 0.26 & 13.268 & 0.233 & 0.0585 & 0.0020 & 0.0752 & 0.0014 & 467.2 \\
\hline 11.1 & 77 & 38 & 0.50 & 5.0 & - & 0.21 & 13.161 & 0.209 & 0.0582 & 0.0017 & 0.0758 & 0.0012 & 471.1 \\
\hline 12.1 & 40 & 12 & 0.31 & 2.6 & 0.000463 & 0.75 & 12.973 & 0.360 & 0.0627 & 0.0025 & 0.0765 & 0.0022 & 475.2 \\
\hline 13.1 & 126 & 82 & 0.65 & 8.2 & - & -0.10 & 13.193 & 0.185 & 0.0557 & 0.0013 & 0.0759 & 0.0011 & 471.4 \\
\hline 14.1 & 89 & 44 & 0.49 & 5.8 & - & 0.36 & 13.212 & 0.204 & 0.0593 & 0.0016 & 0.0754 & 0.0012 & 468.7 \\
\hline 15.1 & 120 & 69 & 0.57 & 7.7 & 0.000740 & 0.39 & 13.389 & 0.199 & 0.0595 & 0.0017 & 0.0744 & 0.0011 & 462.6 \\
\hline 16.1 & 966 & 973 & 1.01 & 25.3 & 0.006747 & 13.03 & 32.790 & 0.376 & 0.1534 & 0.0033 & 0.0265 & 0.0003 & 168.7 \\
\hline 17.1 & 152 & 76 & 0.50 & 9.3 & 0.000109 & 0.37 & 14.110 & 0.193 & 0.0586 & 0.0012 & 0.0706 & 0.0010 & 439.9 \\
\hline 17.2 & 271 & 256 & 0.95 & 16.8 & - & 0.12 & 13.825 & 0.168 & 0.0569 & 0.0009 & 0.0723 & 0.0009 & 449.7 \\
\hline 18.1 & 57 & 17 & 0.29 & 3.5 & 0.000939 & 0.33 & 14.072 & 0.253 & 0.0584 & 0.0021 & 0.0708 & 0.0013 & 441.1 \\
\hline 18.2 & 46 & 17 & 0.36 & 3.1 & 0.000632 & 1.70 & 12.749 & 0.210 & 0.0705 & 0.0018 & 0.0771 & 0.0013 & 478.8 \\
\hline 19.1 & 45 & 17 & 0.38 & 2.9 & 0.000751 & 0.05 & 13.312 & 0.239 & 0.0568 & 0.0021 & 0.0751 & 0.0014 & 466.7 \\
\hline 19.2 & 75 & 42 & 0.56 & 4.7 & 0.000590 & 0.73 & 13.628 & 0.195 & 0.0619 & 0.0013 & 0.0728 & 0.0011 & 453.3 \\
\hline 20.1 & 117 & 57 & 0.49 & 7.8 & 0.000374 & 0.38 & 12.889 & 0.167 & 0.0598 & 0.0012 & 0.0773 & 0.0010 & 480.0 \\
\hline 21.1 & 104 & 66 & 0.63 & 6.2 & 0.000338 & 1.52 & 14.568 & 0.191 & 0.0675 & 0.0016 & 0.0676 & 0.0009 & 421.7 \\
\hline 22.1 & 174 & 134 & 0.77 & 10.5 & 0.000258 & 0.80 & 14.286 & 0.186 & 0.0619 & 0.0010 & 0.0694 & 0.0009 & 432.8 \\
\hline 23.1 & 207 & 132 & 0.64 & 13.5 & 0.000091 & 0.27 & 13.200 & 0.155 & 0.0586 & 0.0009 & 0.0756 & 0.0009 & 469.5 \\
\hline 24.1 & 141 & 82 & 0.58 & 8.8 & 0.000219 & 0.67 & 13.643 & 0.171 & 0.0615 & 0.0010 & 0.0728 & 0.0009 & 453.0 \\
\hline
\end{tabular}

Uncertainties given at the $1 \sigma$ level. Error in AS3 reference zircon calibration was 0.35 and $0.62 \% \%$ for the analytical sessions (not included in above errors but required when comparing data from different mounts). $f_{206} \%$ denotes the percentage of ${ }^{206} \mathrm{~Pb}$ that is common $\mathrm{Pb}$. Correction for common $\mathrm{Pb}$ made using the measured ${ }^{238} \mathrm{U} /{ }^{206} \mathrm{~Pb}$ and ${ }^{207} \mathrm{~Pb} /{ }^{206} \mathrm{~Pb}$ ratios following Tera and Wasserburg (1972) as outlined in Williams (1998). 

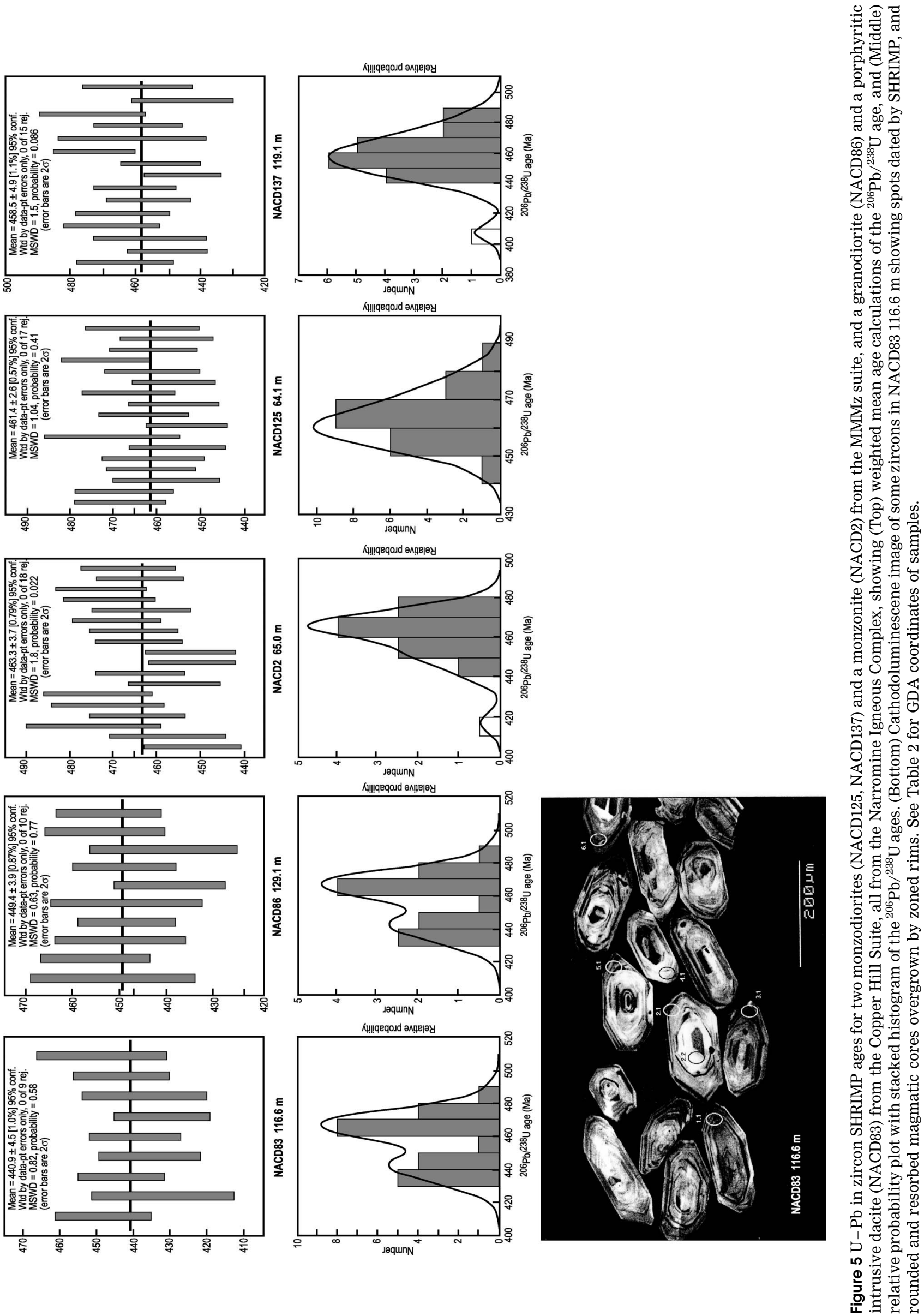

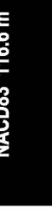


A number of compositional features of the Copper Hill Suite invite comparison with Cenozoic adakites. These include the absence of compositions more mafic than $67 \% \quad \mathrm{SiO}_{2}$ and the strikingly high $\mathrm{Sr} / \mathrm{Y}$ values for such felsic magmas (80-95: Table 3). However, a characteristic feature of adakitic dacites is their notable HREE depletion (Defant \& Drummond 1990), and this feature is not shared by the Copper Hill Suite rocks (Figure $7 \mathrm{~b}$ ), including those in the Narromine Igneous Complex, and elsewhere in the disrupted Macquarie Arc. The high Sr-low Y characteristics of the Copper Hill Suite rocks in the Narromine Igneous Complex are more reasonably accounted for by significant apatite-hornblende fractionation from parental magmas with very high $\mathrm{Sr}$ contents.

A dacite and a granodiorite from the Narromine Igneous Complex Copper Hill Suite have initial $\varepsilon_{\mathrm{Nd}}$ values of +6.4 and +6.9 , respectively (Crawford et al. 2007), little different from that of depleted mantle at $445 \mathrm{Ma}$, indicating minimal involvement of any component derived from ancient continental crust in the genesis of this suite. Further discussion of the petrogenesis of the Copper Hill Suite follows presentation of data for intrusive rocks in the Cowal Igneous Complex.

Table 3 Whole-rock major- and trace-element data (recalculated to $100 \%$ volatile-free) for Narromine Igneous Complex intrusive holocrystalline rocks of the Monzogabbro-Monzodiorite-Monzonite Suite, and intrusive dacite porphyries and granodiorites of the Copper Hill Suite.

\begin{tabular}{|c|c|c|c|c|c|c|c|c|c|c|c|}
\hline \multirow{3}{*}{$\begin{array}{l}\text { Sample no. } \\
\text { Suite } \\
\text { Easting } \\
\text { Northing }\end{array}$} & \multicolumn{11}{|c|}{ Monzogabbro-Monzodiorite-Monzonite Suite } \\
\hline & 597873 & 598617 & 598362 & 597613 & 597853 & 597853 & 595713 & 597113 & 601033 & 603113 & 601113 \\
\hline & 6414060 & 6418184 & 6418167 & 6419184 & 6417184 & 6417184 & 6418484 & 6419184 & 6413163 & 6418184 & 6412184 \\
\hline $\mathrm{SiO}_{2}$ & 58.17 & 51.03 & 66.52 & 59.23 & 49.33 & 50.60 & 49.62 & 55.32 & 60.37 & 58.62 & 61.53 \\
\hline $\mathrm{TiO}_{2}$ & 0.79 & 1.02 & 0.53 & 0.72 & 0.65 & 0.74 & 0.78 & 0.90 & 0.88 & 0.71 & 0.52 \\
\hline $\mathrm{Al}_{2} \mathrm{O}_{3}$ & 15.92 & 18.37 & 15.11 & 19.28 & 19.43 & 17.31 & 18.72 & 17.29 & 17.80 & 19.24 & 18.08 \\
\hline $\mathrm{Fe}_{2} \mathrm{O}_{3}$ & 9.03 & 11.34 & 5.00 & 7.17 & 9.96 & 10.66 & 10.13 & 9.43 & 7.55 & 6.32 & 6.12 \\
\hline $\mathrm{MnO}$ & 0.10 & 0.30 & 0.29 & 0.20 & 0.17 & 0.18 & 0.40 & 0.27 & 0.19 & 0.08 & 0.22 \\
\hline $\mathrm{MgO}$ & 3.00 & 4.38 & 1.99 & 4.80 & 6.10 & 6.69 & 6.60 & 3.77 & 2.30 & 1.78 & 2.47 \\
\hline $\mathrm{CaO}$ & 4.82 & 7.10 & 5.62 & 2.83 & 10.74 & 9.88 & 9.50 & 7.52 & 2.89 & 4.56 & 3.21 \\
\hline $\mathrm{Na}_{2} \mathrm{O}$ & 5.62 & 4.47 & 3.14 & 3.18 & 3.05 & 3.30 & 2.94 & 3.27 & 4.81 & 5.12 & 6.82 \\
\hline $\mathrm{K}_{2} \mathrm{O}$ & 2.09 & 1.45 & 1.67 & 2.33 & 0.43 & 0.42 & 1.04 & 1.83 & 2.77 & 3.30 & 0.82 \\
\hline $\mathrm{P}_{2} \mathrm{O}_{5}$ & 0.45 & 0.54 & 0.14 & 0.26 & 0.15 & 0.21 & 0.27 & 0.40 & 0.45 & 0.28 & 0.21 \\
\hline LOI & 1.88 & 2.33 & 4.34 & 4.32 & 1.51 & 1.50 & 2.63 & 1.88 & 3.75 & 1.65 & 2.29 \\
\hline $\mathrm{Ni}$ & 3 & 7 & 4 & 7 & 32 & 34 & 18 & 8 & 2 & 3 & 7 \\
\hline $\mathrm{Cr}$ & 3 & 13 & 7 & 8 & 66 & 69 & 88 & 8 & 2 & 3 & 7 \\
\hline V & 134 & 333 & 91 & 204 & 263 & 319 & 256 & 219 & 122 & 136 & 145 \\
\hline $\mathrm{Sc}$ & 22 & 24 & 9 & 16 & 25 & 25 & 31 & 20 & 19 & 13 & 9 \\
\hline $\mathrm{Zr}$ & 163 & 47 & 212 & 117 & 19 & 35 & 52 & 152 & 171 & 141 & 93 \\
\hline $\mathrm{Nb}$ & 8.3 & 2.5 & 12.1 & 6.8 & 2 & 3 & 3 & 9 & 7.1 & 8 & 5.3 \\
\hline $\mathrm{Y}$ & 34 & 15 & 22 & 20 & 9 & 11 & 17 & 25 & 26 & 21 & 12 \\
\hline $\mathrm{Sr}$ & 524 & 1053 & 539 & 755 & 1121 & 912 & 716 & 862 & 842 & 918 & 713 \\
\hline $\mathrm{Rb}$ & 18 & 22 & 29 & 41 & 4 & 4 & 23 & 25 & 43 & 50 & 9 \\
\hline $\mathrm{Ba}$ & 386 & 339 & 186 & 323 & 121 & 154 & 82 & 284 & 705 & 373 & 144 \\
\hline $\mathrm{Pb}$ & 2.1 & 3.4 & 3.8 & 4.8 & 1.6 & 2.3 & 3.7 & 5.4 & 5.8 & 4.7 & 22.6 \\
\hline $\mathrm{Zn}$ & 55 & 135 & 148 & 158 & 108 & 83 & 168 & 179 & 131 & 66 & 171 \\
\hline $\mathrm{Cu}$ & 14 & 284 & 5 & 309 & 85 & 95 & 65 & 215 & 87 & 335 & 28 \\
\hline$L a$ & 18.51 & 13.20 & 19.94 & 14.24 & - & - & - & - & 13.12 & - & 10.71 \\
\hline $\mathrm{Ce}$ & 39.52 & 29.30 & 40.22 & 30.33 & - & - & - & - & 25.61 & - & 22.10 \\
\hline$P r$ & 5.23 & 4.25 & 5.08 & 4.08 & - & - & - & - & 3.14 & - & 2.89 \\
\hline$N d$ & 22.77 & 19.77 & 20.51 & 17.7 & - & - & - & - & 12.46 & - & 12.24 \\
\hline$S m$ & 6.02 & 4.95 & 4.44 & 4.22 & - & - & - & - & 2.71 & - & 2.87 \\
\hline$E u$ & 1.60 & 1.95 & 1.21 & 1.57 & - & - & - & - & 0.82 & - & 0.95 \\
\hline$G d$ & 6.23 & 4.37 & 4.07 & 4.18 & - & - & - & - & 2.30 & - & 2.63 \\
\hline$T b$ & 1.04 & 0.59 & 0.65 & 0.64 & - & - & - & - & 0.36 & - & 0.41 \\
\hline Dy & 6.18 & 3.05 & 3.71 & 3.7 & - & - & - & - & 2.03 & - & 2.24 \\
\hline Ho & 1.29 & 0.58 & 0.77 & 0.76 & - & - & - & - & 0.41 & - & 0.46 \\
\hline$E r$ & 3.65 & 1.46 & 2.27 & 2.14 & - & - & - & - & 1.23 & - & 1.31 \\
\hline$Y b$ & 3.34 & 1.18 & 2.36 & 1.97 & - & - & - & - & 1.22 & - & 1.23 \\
\hline$L u$ & 0.51 & 0.18 & 0.37 & 0.3 & - & - & - & - & 0.18 & - & 0.19 \\
\hline$H f$ & 4.26 & 1.30 & 4.97 & 2.71 & - & - & - & - & 3.14 & - & 2.33 \\
\hline$T a$ & 0.45 & 0.14 & 0.81 & 0.43 & - & - & - & - & 0.48 & - & 0.33 \\
\hline$T h$ & 2.99 & 0.99 & 3.75 & 1.60 & - & - & - & - & 2.14 & - & 1.35 \\
\hline$U$ & 1.20 & 0.49 & 1.83 & 0.96 & - & - & - & - & 1.06 & - & 0.62 \\
\hline
\end{tabular}


Table 3 (Continued)

\begin{tabular}{|c|c|c|c|c|c|c|c|c|c|c|c|}
\hline \multirow{3}{*}{$\begin{array}{l}\text { Sample no. } \\
\text { Suite } \\
\text { Easting } \\
\text { Northing }\end{array}$} & \multicolumn{11}{|c|}{ Monzogabbro-Monzodiorite-Monzonite Suite } \\
\hline & 604113 & 603613 & 603613 & 603613 & 604113 & 604613 & 604613 & 603113 & 603613 & 604113 & 602613 \\
\hline & 6417184 & 6418184 & 6417734 & 6417734 & 6417684 & 6417184 & 6417684 & 6417684 & 6418684 & 6418684 & 6418684 \\
\hline $\mathrm{SiO}_{2}$ & 54.03 & 55.67 & 50.37 & 51.24 & 58.32 & 56.04 & 57.00 & 54.43 & 55.04 & 56.02 & 59.32 \\
\hline $\mathrm{TiO}_{2}$ & 0.91 & 0.75 & 0.93 & 1.00 & 0.58 & 0.89 & 0.63 & 0.82 & 0.83 & 0.71 & 0.64 \\
\hline $\mathrm{Al}_{2} \mathrm{O}_{3}$ & 17.79 & 18.55 & 17.49 & 17.44 & 18.63 & 17.78 & 19.91 & 18.60 & 18.16 & 18.00 & 19.21 \\
\hline $\mathrm{Fe}_{2} \mathrm{O}_{3}$ & 9.24 & 7.47 & 10.83 & 11.02 & 6.73 & 8.40 & 6.18 & 8.59 & 8.65 & 7.94 & 5.24 \\
\hline $\mathrm{MnO}$ & 0.20 & 0.18 & 0.22 & 0.20 & 0.15 & 0.18 & 0.14 & 0.17 & 0.21 & 0.17 & 0.15 \\
\hline $\mathrm{MgO}$ & 3.90 & 2.61 & 4.92 & 4.75 & 3.25 & 3.08 & 2.45 & 3.31 & 3.76 & 3.12 & 2.06 \\
\hline $\mathrm{CaO}$ & 6.99 & 8.12 & 8.19 & 9.15 & 5.36 & 6.26 & 5.09 & 7.16 & 5.16 & 6.29 & 3.90 \\
\hline $\mathrm{Na}_{2} \mathrm{O}$ & 4.38 & 4.74 & 4.25 & 3.77 & 5.05 & 4.63 & 5.76 & 4.67 & 5.32 & 4.99 & 6.53 \\
\hline $\mathrm{K}_{2} \mathrm{O}$ & 2.04 & 1.57 & 1.97 & 1.11 & 1.69 & 2.26 & 2.46 & 1.77 & 2.47 & 2.46 & 2.63 \\
\hline $\mathrm{P}_{2} \mathrm{O}_{5}$ & 0.52 & 0.35 & 0.84 & 0.33 & 0.24 & 0.47 & 0.37 & 0.47 & 0.40 & 0.30 & 0.32 \\
\hline LOI & 1.79 & 2.12 & 2.48 & 1.36 & 2.74 & 1.51 & 2.17 & 2.00 & 2.90 & 1.83 & 3.50 \\
\hline $\mathrm{Ni}$ & 6 & 4 & 7 & 8 & 9 & 6 & 2 & 6 & 4 & 3 & 2 \\
\hline $\mathrm{Cr}$ & 11 & 5 & 8 & 8 & 22 & 10 & 4 & 14 & 5 & 4 & 2 \\
\hline $\mathrm{V}$ & 269 & 198 & 365 & 402 & 165 & 218 & 154 & 285 & 258 & 218 & 120 \\
\hline Sc & 18 & 16 & 21 & 25 & 10 & 18 & 12 & 20 & 18 & 17 & 12 \\
\hline $\mathrm{Zr}$ & 149 & 107 & 75 & 83 & 96 & 71 & 127 & 90 & 110 & 123 & 164 \\
\hline $\mathrm{Nb}$ & 5.8 & 6 & 4 & 4 & 6 & 7 & 6.0 & 4.7 & 5 & 6 & 7 \\
\hline $\mathrm{Y}$ & 19 & 18 & 17 & 15 & 12 & 20 & 16 & 18 & 18 & 17 & 17 \\
\hline $\mathrm{Sr}$ & 1132 & 1101 & 1115 & 1094 & 933 & 855 & 1009 & 1032 & 908 & 881 & 555 \\
\hline $\mathrm{Rb}$ & 33 & 20 & 32 & 14 & 27 & 31 & 37 & 21 & 37 & 36 & 36 \\
\hline $\mathrm{Ba}$ & 675 & 209 & 257 & 208 & 339 & 316 & 417 & 329 & 395 & 309 & 313 \\
\hline $\mathrm{Pb}$ & 5.8 & 6.2 & 2.2 & 2.7 & 1.8 & 3.9 & 3.8 & 4.7 & 2.6 & 2.7 & 3.3 \\
\hline $\mathrm{Zn}$ & 108 & 79 & 104 & 99 & 99 & 91 & 72 & 104 & 87 & 93 & 80 \\
\hline $\mathrm{Cu}$ & 337 & 118 & 559 & 587 & 113 & 332 & 96 & 268 & 215 & 111 & 114 \\
\hline$L a$ & 19.30 & - & - & - & - & - & 20.05 & 18.40 & - & - & - \\
\hline $\mathrm{Ce}$ & 42.31 & - & - & - & - & - & 43.32 & 39.74 & - & - & - \\
\hline$P r$ & 5.89 & - & - & - & - & - & 5.9 & 5.43 & - & - & - \\
\hline$N d$ & 25.8 & - & - & - & - & - & 24.84 & 23.72 & - & - & - \\
\hline$S m$ & 5.89 & - & - & - & - & - & 5.81 & 5.36 & - & - & - \\
\hline$E u$ & 1.76 & - & - & - & - & - & 1.75 & 1.69 & - & - & - \\
\hline$G d$ & 5.05 & - & - & - & - & - & 4.77 & 4.56 & - & - & - \\
\hline$T b$ & 0.71 & - & - & - & - & - & 0.67 & 0.64 & - & - & - \\
\hline$D y$ & 3.71 & - & - & - & - & - & 3.53 & 3.42 & - & - & - \\
\hline Ho & 0.71 & - & - & - & - & - & 0.68 & 0.65 & - & - & - \\
\hline$E r$ & 1.89 & - & - & - & - & - & 1.86 & 1.77 & - & - & - \\
\hline$Y b$ & 1.66 & - & - & - & - & - & 1.64 & 1.58 & - & - & - \\
\hline$L u$ & 0.24 & - & - & - & - & - & 0.24 & 0.23 & - & - & - \\
\hline$H f$ & 2.68 & - & - & - & - & - & 2.58 & 2.49 & - & - & - \\
\hline$T a$ & 0.34 & - & - & - & - & - & 0.22 & 0.25 & - & - & - \\
\hline$T h$ & 2.56 & - & - & - & - & - & 1.84 & 2.02 & - & - & - \\
\hline$U$ & 1.28 & - & - & - & - & - & 0.87 & 0.77 & - & - & - \\
\hline
\end{tabular}

(continued)

\section{Alteration of the Narromine Igneous Complex Copper Hill Suite dacites and granodiorites}

Weakly developed $\mathrm{Cu}-\mathrm{Au}$ mineralisation occurs in the central section of the western Narromine Igneous Complex (Cooke et al. 2007). Mineralisation is spatially (and genetically?) associated with the intrusive dacites granodiorites of the Copper Hill Suite, which has intruded the MMMz suite rocks, also shown in drillholes NACD 8, 83, 89 and 91. A propylitic alteration assemblage (epidote-chlorite-carbonate-quartz-sericite) is developed in the dacitic and granodioritic rocks. $\mathrm{Cu}-$ $\mathrm{Au}$ mineralisation occurs primarily as quartz-magnetite-epidote-chlorite-chalcopyrite veins, and also as minor disseminations in propylitic-altered rocks. The dacites have also undergone localised intense sericite- carbonate-pyrite-(chlorite) alteration near major faults. However, this hydrothermal activity is not associated with any known mineralisation. Significant hydrothermal alteration is indicated by occasional adularia veinlets in drillhole NACD68 (at $98.5 \mathrm{~m}$ ).

Calculated temperatures of formation for the propylitic alteration assemblage and related quartz - epidotemagnetite-chalcopyrite veins are between 350 and $450^{\circ} \mathrm{C}$, based on O-isotope results and application of the quartz-epidote geothermometer. Hypersaline fluid inclusions have been observed locally in quartz phenocrysts and quartz-epidote veins, indicating the presence of magmatic-hydrothermal fluids typical of porphyry-style mineralisation. Apart from the local intensive development of 'epidosite', and the aforementioned barren sericitic alteration, alteration 
Table 3 (Continued).

\begin{tabular}{|c|c|c|c|c|c|c|c|}
\hline $\begin{array}{l}\text { Sample no. } \\
\text { Suite }\end{array}$ & $82-120.0$ & 83-116.6 & 83-148.4 & $\begin{array}{l}\text { 89-137.2 } \\
\text { Copper Hill Suite }\end{array}$ & $8-137.7$ & 86-129.1 & $137-119.1$ \\
\hline Easting & 598617 & 598114 & 598114 & 597613 & 598027 & 598004 & 598113 \\
\hline Northing & 6418184 & 6417687 & 6417687 & 6419184 & 6418273 & 6418437 & 6416734 \\
\hline $\mathrm{SiO}_{2}$ & 70.01 & 67.58 & 68.53 & 69.99 & 69.39 & 68.05 & 66.67 \\
\hline $\mathrm{TiO}_{2}$ & 0.26 & 0.26 & 0.26 & 0.30 & 0.26 & 0.29 & 0.31 \\
\hline $\mathrm{Al}_{2} \mathrm{O}_{3}$ & 16.14 & 15.81 & 16.28 & 16.72 & 15.95 & 16.50 & 17.20 \\
\hline $\mathrm{Fe}_{2} \mathrm{O}_{3}$ & 3.04 & 5.30 & 3.55 & 2.75 & 3.31 & 4.29 & 4.32 \\
\hline $\mathrm{MnO}$ & 0.09 & 0.04 & 0.08 & 0.04 & 0.11 & 0.06 & 0.23 \\
\hline MgO & 1.29 & 1.09 & 1.14 & 2.02 & 1.33 & 1.38 & 1.69 \\
\hline $\mathrm{CaO}$ & 1.68 & 2.85 & 2.74 & 1.55 & 1.79 & 2.68 & 1.89 \\
\hline $\mathrm{Na}_{2} \mathrm{O}$ & 5.28 & 4.77 & 5.10 & 4.97 & 4.51 & 4.54 & 5.22 \\
\hline $\mathrm{K}_{2} \mathrm{O}$ & 2.12 & 2.18 & 2.23 & 1.56 & 3.28 & 2.10 & 2.37 \\
\hline $\mathrm{P}_{2} \mathrm{O}_{5}$ & 0.09 & 0.11 & 0.09 & 0.09 & 0.09 & 0.11 & 0.10 \\
\hline LOI & 2.16 & 0.97 & 1.79 & 2.84 & 1.79 & 1.61 & 2.01 \\
\hline $\mathrm{Ni}$ & 5 & 5 & 7 & bdl & 9 & 7 & 7 \\
\hline $\mathrm{Cr}$ & 22 & 20 & 23 & 22 & 24 & 25 & 31 \\
\hline V & 55 & 54 & 57 & 86 & 52 & 57 & 68 \\
\hline $\mathrm{Sc}$ & 4 & 5 & 5 & 7 & 4 & 5 & 4 \\
\hline $\mathrm{Zr}$ & 98 & 103 & 97 & 102 & 98 & 97 & 100 \\
\hline $\mathrm{Nb}$ & 5 & 6 & 5.7 & 6.5 & 6.7 & 6.0 & 5.9 \\
\hline $\mathrm{Y}$ & 8 & 8 & 8 & 9 & 7 & 8 & 7 \\
\hline $\mathrm{Sr}$ & 593 & 718 & 659 & 728 & 571 & 762 & 541 \\
\hline $\mathrm{Rb}$ & 26 & 23 & 27 & 0 & 43 & 22 & 37 \\
\hline $\mathrm{Ba}$ & 524 & 447 & 511 & 207 & 650 & 480 & 538 \\
\hline $\mathrm{Pb}$ & 8.8 & 4.8 & 7.0 & 1.8 & 4.6 & 4.3 & 4.8 \\
\hline $\mathrm{Zn}$ & 71 & 44 & 51 & 0 & 100 & 80 & 193 \\
\hline $\mathrm{Cu}$ & 249 & 408 & 96 & 0 & 1542 & 73 & 15 \\
\hline$L a$ & - & - & 10.33 & 16.62 & 12.20 & 9.52 & 8.30 \\
\hline $\mathrm{Ce}$ & - & - & 18.24 & 30.94 & 21.41 & 17.30 & 15.83 \\
\hline $\mathrm{Pr}$ & - & - & 2.08 & 3.67 & 2.27 & 1.98 & 1.88 \\
\hline$N d$ & - & - & 7.97 & 14.33 & 7.91 & 7.33 & 7.34 \\
\hline$S m$ & - & - & 1.63 & 2.80 & 1.42 & 1.51 & 1.50 \\
\hline Eu & - & - & 0.57 & 1.22 & 0.39 & 0.52 & 0.48 \\
\hline$G d$ & - & - & 1.39 & 2.41 & 1.20 & 1.30 & 1.25 \\
\hline$T b$ & - & - & 0.22 & 0.34 & 0.18 & 0.21 & 0.19 \\
\hline Dy & - & - & 1.25 & 1.98 & 1.04 & 1.2 & 1.14 \\
\hline Ho & - & - & 0.26 & 0.41 & 0.22 & 0.26 & 0.24 \\
\hline$E r$ & - & - & 0.78 & 1.18 & 0.70 & 0.76 & 0.76 \\
\hline$Y b$ & - & - & 0.85 & 1.13 & 0.81 & 0.88 & 0.81 \\
\hline$L u$ & - & - & 0.14 & 0.17 & 0.14 & 0.15 & 0.13 \\
\hline$H f$ & - & - & 2.44 & 2.48 & 2.52 & 2.59 & 2.32 \\
\hline$T a$ & - & - & 0.39 & 0.43 & 0.56 & 0.45 & 0.42 \\
\hline$T h$ & - & - & 1.76 & 1.90 & 2.68 & 1.93 & 1.17 \\
\hline$U$ & - & - & 1.27 & 1.05 & 3.03 & 0.81 & 0.57 \\
\hline
\end{tabular}

Trace elements in italics measured by ICP-MS, remainder and majors by XRF. bdl, below detection limit. See Appendix 1 for rock types analysed.

assemblages intersected during drilling of the Narromine Igneous Complex are only weakly developed, indicating low fluid fluxes and limited mineral potential. Magnetite-destructive alteration zones appear not to be prospective for copper - gold mineralisation, based on the material intersected to date.

\section{COWAL AND FAIRHOLME IGNEOUS COMPLEXES}

South of Forbes, the Junee-Narromine Volcanic Belt is defined by a number of unexposed but aeromagnetically defined bodies, including the Cowal Igneous
Complex east of the Booberoi Shear Zone and the Fairholme Igneous Complex west of the fault (Figure 8). North Ltd, and more recently Barrick Gold Ltd, carried out extensive exploration in the Cowal Igneous Complex (Miles \& Brooker 1998; Bywater et al. 2004) with widespread aircore programs that in many instances have yielded good rock samples from diamond tails. Specific target areas recognised as Endeavour (E)35, E39, E41, E42 and E43 cover a large part of the central and western part of the Cowal Igneous Complex (Figure 8), and a few diamond drillholes in the northern part (LCD12, LCD13) provided further material for study. E42 is in development to be a significant gold mine with an estimated resource of $63.6 \mathrm{Mt} @ 1.22 \mathrm{~g} / \mathrm{t}$ 

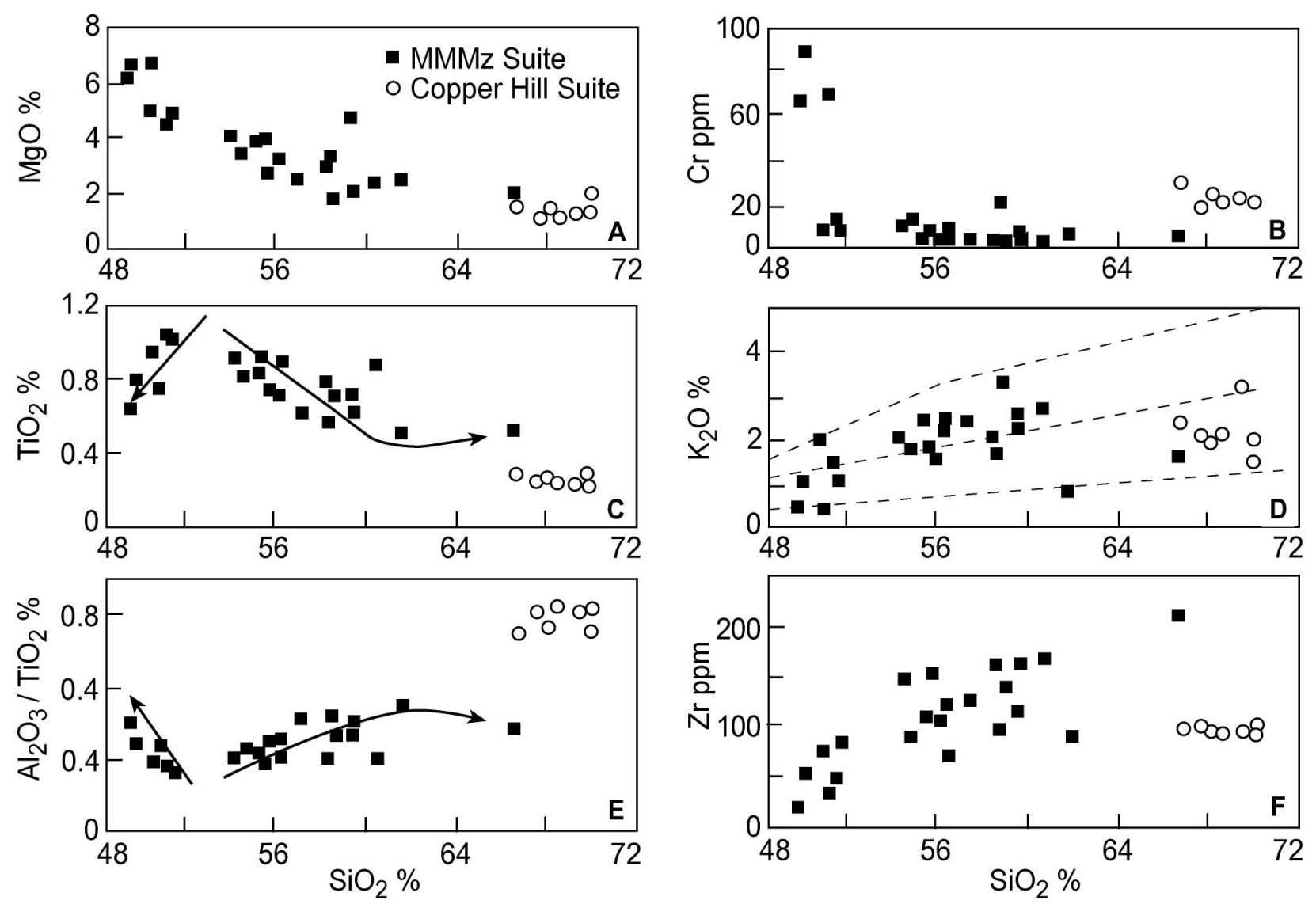

Figure 6 Some major and trace elements $v s \mathrm{SiO}_{2}$ for intrusive rocks from the Narromine Igneous Complex, chosen to show distinct compositional fields for the MMMz suite compared to the granodioritic and dacitic-rhyolitic rocks assigned to the Phase 3 Copper Hill Suite. Arrows in the MMMz suite show trends for accumulation of a plagioclase-clinopyroxenehornblende-dominant assemblage $\left(<52 \% \mathrm{SiO}_{2}\right)$ and an interpreted liquid line of descent $\left(>52 \% \mathrm{SiO}_{2}\right)$.

gold (Bywater et al. 2004). Newcrest's Marsden prospect, close to the southeastern faulted margin of the Cowal Igneous Complex (Figure 8), has been the subject of ongoing exploration, and Newcrest also carried out drilling at several prospects in the Fairholme Igneous Complex.

\section{Cowal Igneous Complex}

Due to complete burial by much younger cover, including the Quaternary sediments of Lake Cowal, the only part of the Cowal Igneous Complex in which a lithostratigraphy (albeit of limited extent and thickness) has been determined is around E42 (the Cowal gold deposit), where a sequence of trachytic lavas and volcaniclastics has been recognised (Miles \& Brooker 1998). These rocks, including the main volcanic unit, the Golden Lava, have been intruded by a major dioritic intrusion, the Muddy Lake Gabbro, for which available radiometric dates suggest a Middle Ordovician age (465-456 Ma: see below). Interpretation of aeromagnetic data (Figure 1) by exploration groups, groundtruthed by aircore sampling, suggests that much of the southern Cowal Igneous Complex may be made up of Ordovician intrusive rocks, with at least one high-level granodioritic intrusion known from drillholes LCD12 and
13 in the northern part of the complex. Eastonian limestone is known from the Marsden area, but its relationships with the adjacent rocks remain unknown (Percival \& Glen 2007).

\section{VOLCANIC AND VOLCANICLASTIC ROCKS}

Away from E42, lavas are poorly represented in the Cowal Igneous Complex. Two analyses of lavas, one andesitic, the other basaltic, from E46 (Figure 8), were reported by Bastrakov (1998). Lavas are also present in drillholes around E43, most being of trachyandesite to trachyte compositions with phenocrysts of plagioclase, augite, FeTi oxides and apatite microphenocrysts. Formerly glassy textures are common. Four lavas from the E43 prospect west of Marsden have been analysed (Table 4: see Appendix 1 for details of rock types analysed), and these range from 55 to $67 \% \mathrm{SiO}_{2}$.

Lavas are well represented from the E42 area drilling, with nine samples of the Golden Lava trachyandesites-trachytes chosen for whole-rock analysis (Table 4). These lavas are all formerly glassy, often spherulitic or banded felsic lavas with sparse plagioclase phenocrysts, rare chloritised mafic phenocrysts, and common apatite microphenocrysts. They are best classified as trachyandesites to trachytes, with $61-67 \%$ $\mathrm{SiO}_{2}$, a range probably exaggerated by $\mathrm{SiO}_{2}$ mobility 
Figure 7 Chondrite-normalised REE patterns for (a) a monzogabbro and two monzodiorites from the MMMz suite, and (b) granodiorites and intrusive dacite porphyries from the Copper Hill Suite, both from the Narromine Igneous Complex. (c) N-MORB normalised multi-element patterns for an average $\mathrm{MMMz}$ suite monzodiorite compared with a typical Fijian shoshonite lava and an average Walli Volcanics basalt from the Molong Volcanic Belt (standardising data from Sun \& McDonough 1989; data from Crawford et al. 2007).
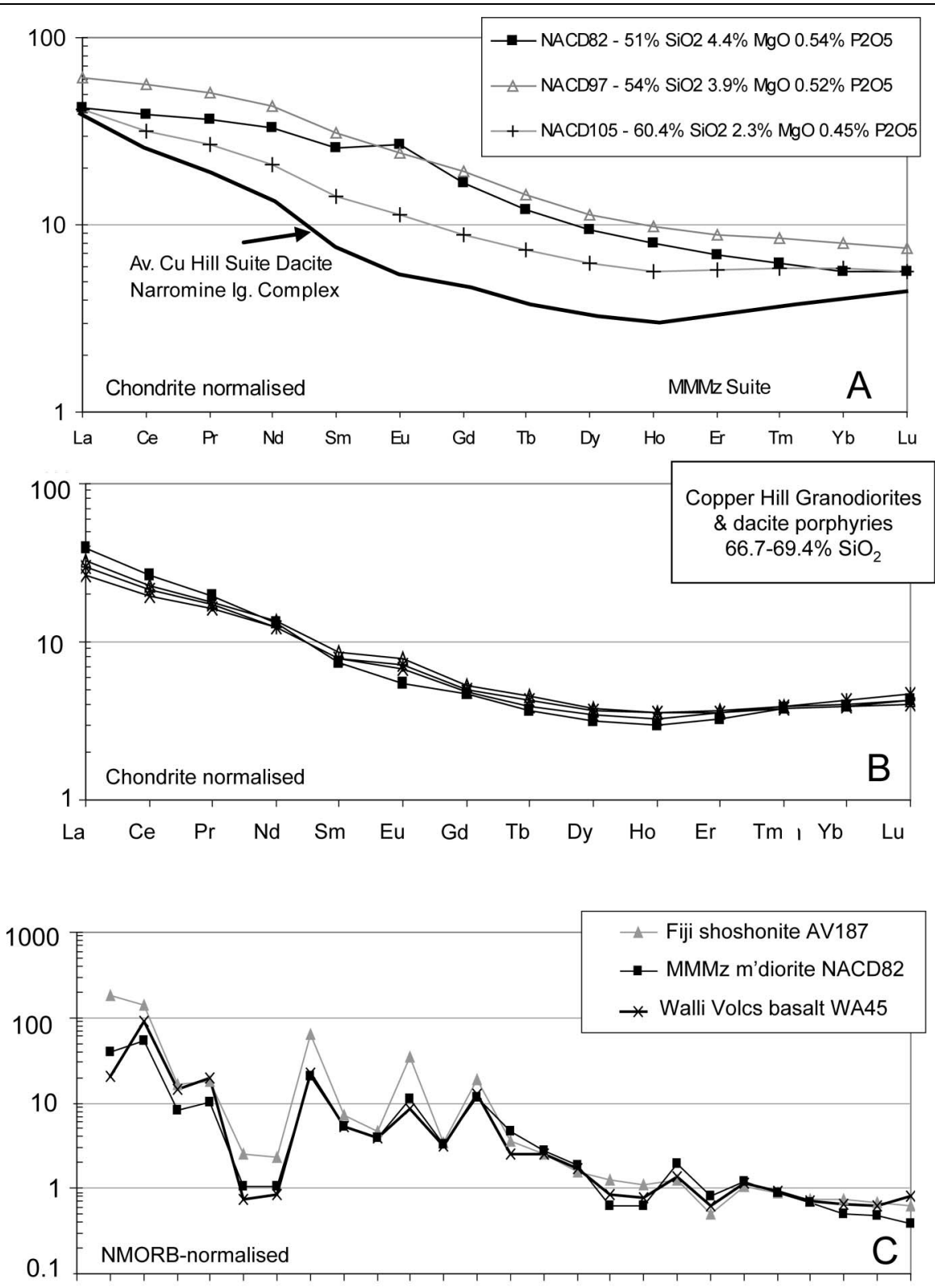

$\mathrm{Rb}$ Ba Th U Nb Ta K La CePb Pr Sr P NdSm Zr Hf Eu Ti Gd Tb Dy Y Er Yb during alteration. The single more mafic rock from E42 classified petrographically as a lava (E42H361-340.6) is a plagioclase+augite-phyric trachyandesite with $52 \%$ $\mathrm{SiO}_{2}$ and $5.5 \% \mathrm{MgO}$, and a REE pattern (Figure $4 \mathrm{~b}$ ) perfectly parallel to those for the felsic lavas, suggesting that is a more mafic comagmatic representative of the same suite (see below).

Geochemical affinities of the Phase 1 Golden Lava trachytes and trachyandesites and associated feeder dykes from $\mathrm{E} 42$ have been described in Glen et al. (2007a). At $62-65 \% \mathrm{SiO}_{2}$, best-preserved lavas have 3.5$4.5 \% \mathrm{~K}_{2} \mathrm{O}$ and $0.40-0.45 \% \mathrm{P}_{2} \mathrm{O}_{5}$, falling in the high- $\mathrm{K}$ calc-alkaline series close to the border with shoshonitic lavas (Figure 6d). The E42 lavas were therefore considered to be more fractionated variants of the same broad high-K calc-alkaline magma type as the Phase 1 Nelungaloo Volcanics west of Parkes, some $50 \mathrm{~km}$ northeast of Cowal.
Only four lavas are recorded by drilling at E43 and $\mathrm{E} 46$, and these vary from trachyandesitic to trachytic compositions. An REE pattern (Figure 4b) for an E43 andesite shows a typical flat-topped Nelungaloo-type pattern, and exactly parallels at expected lower levels the E42 Golden Lava REE patterns. On this basis, the E43 andesites are considered to be part of the same Nelungaloo-type Phase 1 magmatic episode as the E42 trachyandesites. The E43 dacitic/trachytic lavas with $66-67 \% \mathrm{SiO}_{2}$ are probably even more evolved counterparts of the same suite, although no REE data are available to confirm this broad correlation.

Two trachytes (one a formerly glassy lava, the other a feeder dyke to the lavas) from E42, and a trachyandesite lava from $\mathrm{E} 43$ have been analysed for the $\mathrm{Nd}$ isotopic ratios (Crawford et al. 2007). Initial $\varepsilon_{\mathrm{Nd}}$ values for these three samples, all calculated at $475 \mathrm{Ma}$, are +7.8 and +7.9 for the $\mathrm{E} 42$ rocks, and +8.1 for the $\mathrm{E} 43$ lava. These values 


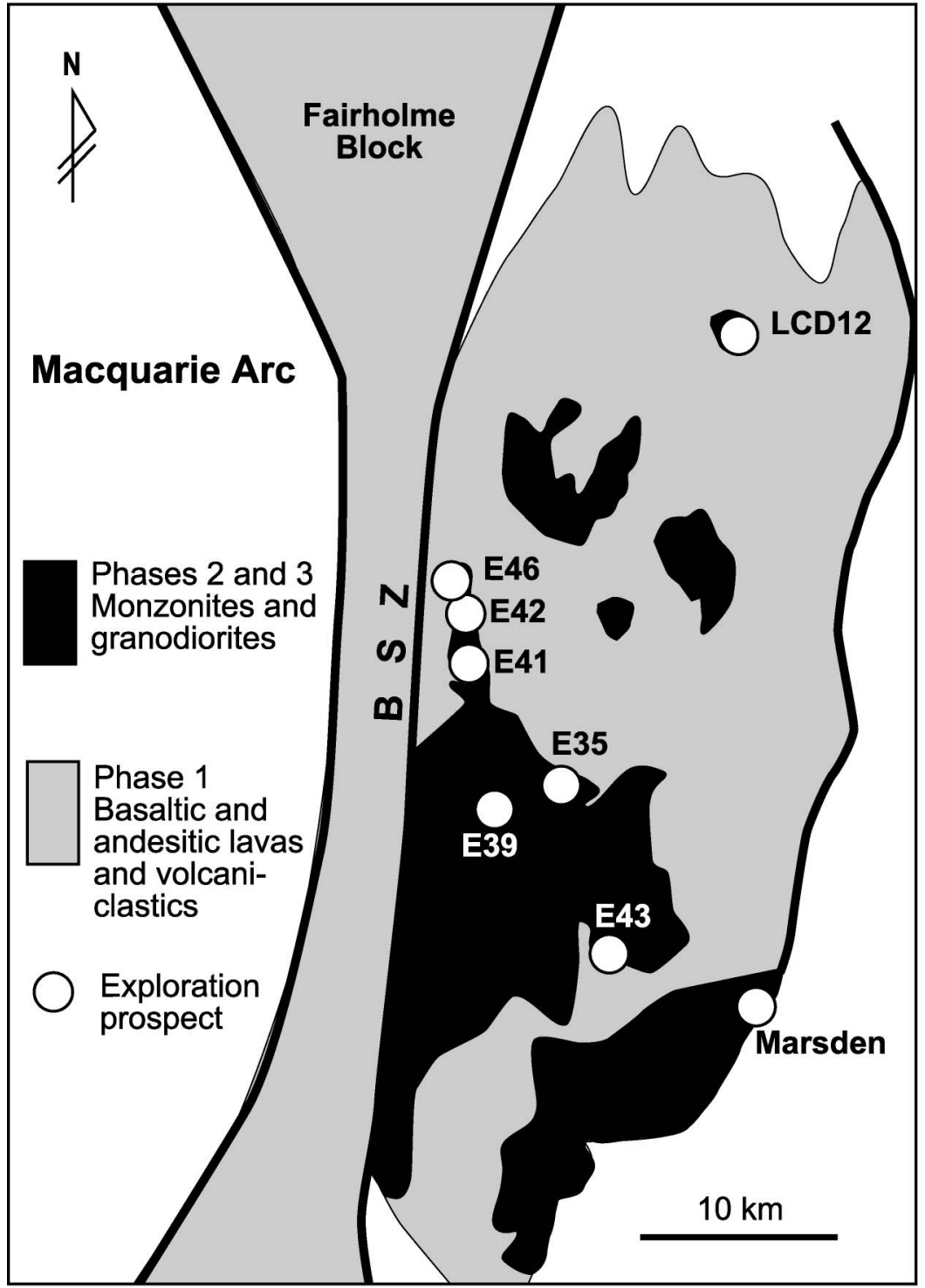

Figure 8 Generalised distribution of the Ordovician rocks and exploration prospects of the JuneeNarromine Volcanic Belt in the Cowal Igneous Complex. BSZ, Booberoi Shear Zone. are slightly higher than data for the Nelungaloo Volcanics (+7.3 and +7.5: Crawford et al. 2007; Glen et al. 2007b) but confirm that, as for the Nelungaloo Volcanics further north in the belt, this Phase 1 magmatism in the Macquarie Arc involved no ancient crustal component, since these values are close to that of the depleted mantle in the Early Ordovician.

\section{INTRUSIVE ROCKS}

The holocrystalline intrusive rocks in the Cowal Igneous Complex show great petrographic and geochemical variation, ranging from mafic gabbros with $10-12 \%$ $\mathrm{MgO}$ from the Marsden drillholes, to high-level granophyric felsic intrusive rocks (granodiorites herein) with $67-76 \% \mathrm{SiO}_{2}$ from several localities. Relatively coarsegrained hornblende gabbros are well represented at Marsden, and cumulate wehrlite (olivine+clinopyroxene) and quite mafic olivine gabbro were drilled at E41. Gabbros and hornblende gabbros grade through mafic diorites to hornblende and quartz diorites or monzodiorites, with common brown to straw magmatic hornblende, not uncommon brownish biotite, and common quartz. Coarse primary sphene is present in a granodiorite from aircore hole 1504/63. Quenched marginal phases of granodioritic composition rocks contain quartz and plagioclase phenocrysts, with subordinate hornblende, and are well represented in drillholes E39/H1 and E39H3, LCD7 around $150 \mathrm{~m}$, and 1504/62. $\mathrm{K}$-feldspar is subordinate to plagioclase in most rocks, but occasional rocks trending to monzonitic compositions are present, and these contain both plagioclase and K-feldspar phenocrysts (e.g. in drillholes 1504/ $17,1504 / 43, \mathrm{E} 35 \mathrm{H} 1$ at $9 \mathrm{~m}, \mathrm{E} 43 / 5$ at $297 \mathrm{~m}$ ). Apatite is most abundant in the mafic and dioritic compositions, occurring as stout prisms, whereas in felsic intrusive rocks it is usually elongate to acicular. Well-formed zircons are not uncommon in the intermediate and felsic intrusive rocks. The most distinctive difference to the MMMz suite intrusive rocks in the Narromine Igneous Complex is the relative abundance of quartz in dioritic and more evolved rocks in the Cowal Igneous Complex.

Our geochronological and geochemical study indicates that at least two major suites of intrusive rocks are 
represented in the Cowal Igneous Complex, and these are very close in age and composition to those described above from the Narromine Igneous Complex. The most significant difference based on available data is that the Copper Hill Suite representatives in the Cowal Igneous Complex extend to more mafic compositions than the dacites and granodiorites in the Narromine Igneous Complex.

Drilling intersected numerous mafic dykes in the Cowal Igneous Complex, most of which transect coarser grained intrusive rocks. The dykes range from distinctive coarsely plagioclase-phyric basalts with abundant sericitised centimetre-long plagioclase phenocrysts common in E42, to aphyric basaltic to microdoleritic rocks. Clinopyroxene-phyric and aphyric basalts are present in most E43 drillholes and post-date the $447 \mathrm{Ma}$ host granodiorite, suggesting correlation with Phase 4 magmatism. At Marsden, both low greenschist facies aphyric basaltic and doleritic dykes occur, as well as much fresher Ti-augite-bearing transitional alkaline dolerite dykes similar to those noted elsewhere in the Macquarie Arc and interpreted as possibly Silurian in age (Crawford et al. 2007).

\section{GEOCHRONOLOGY OF COWAL IGNEOUS COMPLEX INTRUSIVE ROCKS}

Using K-Ar methods, Perkins et al. (1995) constrained the time of intrusion of the Muddy Lake Gabbro to between 465 and $456 \mathrm{Ma}$, and showed that mineralisation-related alteration was significantly younger, around $439 \mathrm{Ma}$. Magmatic hornblende from a granodiorite at E39 was also dated by the same technique and gave an age of $465.7 \pm 1 \mathrm{Ma}$ (Perkins et al. 1995), although SHRIMP U $-\mathrm{Pb}$ zircon dating for the same sample yielded an age of $450.9 \pm 4.5 \mathrm{Ma}$ (Bastrakov 1998). The latter author also recorded an ${ }^{40} \mathrm{Ar}-{ }^{39} \mathrm{Ar}$ age of $466.8 \pm 1.5 \mathrm{Ma}$ on $\mathrm{E} 42$ diorite magmatic hornblende from drillhole $1504 / 13$, yet a SHRIMP U-Pb zircon age from the same sample yielded an age of $455.9 \pm 5.6 \mathrm{Ma}$ (no information was provided on the number of grains analysed to reach the age given). This discrepancy between the $\mathrm{Ar}-\mathrm{Ar}$ ages and the SHRIMP zircon $\mathrm{U}-\mathrm{Pb}$ ages remains unexplained.

Although more geochronological studies of carefully constrained samples are clearly warranted for the Cowal Igneous Complex, the age range for the intrusive rocks matches very well similar MMMz suite intrusive rocks in the Narromine Igneous Complex, and argues for a major regional pulse of mainly high-K magmatism in the Darriwilian (Middle Ordovician). The age of mineralisation at Cowal is remarkably close to that at Northparkes and Cadia (mainly 440-435 Ma: Bastrakov 1998; Lickfold et al. 2003, 2007; Forster et al. 2004; Crawford et al. 2007) for a zero porphyry trachyte dyke that transects mineralisation at E37 in the Northparkes leases). This may imply that the structurally controlled vein-type mineralisation at Cowal E42 is linked to undiscovered $c a 440$ Ma monzonite-type intrusive rocks such as those demonstrably related to the porphyrystyle mineralisation at Northparkes and Cadia, or that deformation at this time as part of the Benambran Orogeny created the veins.

We have used laser ablation ICP-MS U-Pb dating of zircons to date a granodiorite from E43 $\mathrm{H} 4$ at $159.8 \mathrm{~m}$ at
$447 \pm 11 \mathrm{Ma}$ (Table 5; Figure 9). Details of the dating technique are given in Meffre et al. (2007). Although this age is based on only 12 grains, these zircons show bimodal range of dates recorded in the Narromine Igneous Complex Copper Hill Suite rocks. Four zircons have ages around $445 \mathrm{Ma}$, matching the younger population in the Copper Hill Suite rocks from the Narromine Igneous Complex, those in the Molong Volcanic Belt (Crawford et al. 2007), and also the SHRIMP zircon ages noted above from an E39 granodiorite. The older ages range from between 474 and $459 \mathrm{Ma}$, spanning the same age range as zircons in the Narromine Igneous Complex MMMz suite.

In summary, although further dating studies are required, we interpret available data as recording two major magmatic events for intrusive rocks in the Cowal Igneous Complex, a Darriwilian episode ( $c a 465 \mathrm{Ma}$ ) that generated a monzogabbro-monzodiorite suite akin to that in the Narromine Igneous Complex, and a broadly Bolindian-late Eastonian ( $c$ 4 456-445 Ma) hornblen$\mathrm{de}+$ quartz diorite-granodiorite-porphyritic dacite suite correlated with the Copper Hill Suite rocks elsewhere in the Macquarie Arc.

\section{GEOCHEMISTRY OF COWAL IGNEOUS COMPLEX INTRUSIVE ROCKS}

Holocrystalline intrusive rocks from the Cowal Igneous Complex have been geographically grouped into four suites for the purposes of geochemical assessment and presentation. These groups, with reference to the locality map (Figure 8), are: (i) North Cowal Intrusives, all high-level intrusive K-feldspar + quartz-phyric monzogranites with distinctive, almost aplitic textures, and all come from drillhole LCD 12 and adjacent areas, indicating the presence of a distinctive, evolved monzogranite intrusive unit in this area; (ii) Marsden area intrusives comprising rocks from the eastern and southeastern side of the Cowal Igneous Complex, including Newcrest's Marsden prospect, and North's E43 prospect; (iii) a large granodiorite intrusion widespread in drilling around the E39 prospect area, south of E42 gold development; and (iv) intrusives in the E41 and E42 prospect areas that include a range of gabbroic to granodioritic and monzonitic rocks from the western side of the Cowal Igneous Complex and are best represented by samples of Muddy Lake Gabbro at E42.

Samples were carefully selected petrographically to avoid alteration, and are among the freshest rocks analysed from the Macquarie Arc. Geochemical data (representative analyses in Table 4) are compared in Figure 10 with data for the Copper Hill Suite intrusives regionally, for the Phase 4 shoshonitic Cadia and Northparkes intrusives suites, and for the Phase 2 Narromine Igneous Complex MMMz suite. Representative chondrite-normalised REE patterns are given in Figure 11.

\section{North Cowal intrusives}

The North Cowal intrusives form a separate compositional grouping from the other Cowal intrusive suites. 


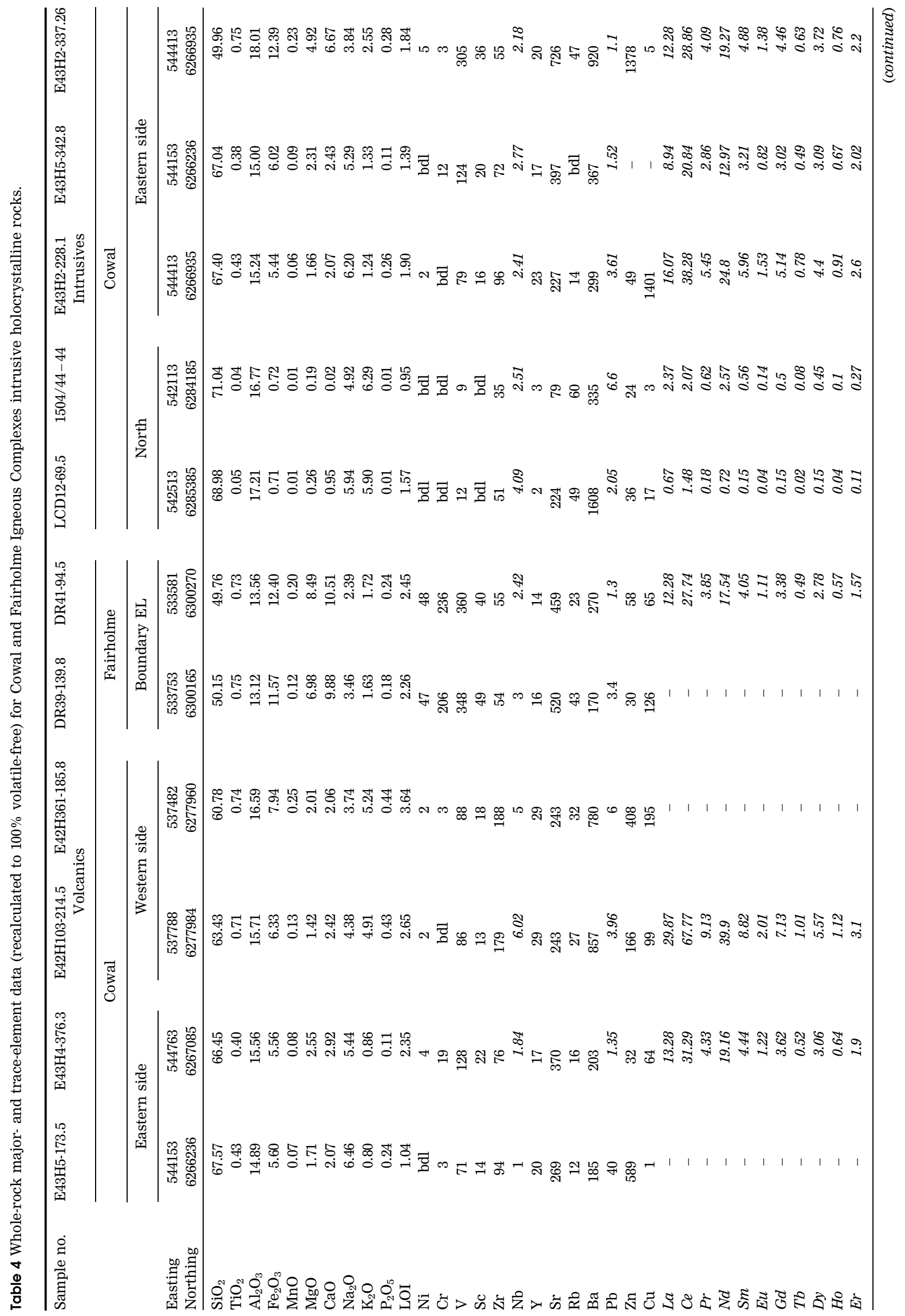


These rocks are monzogranites with around $70 \% \mathrm{SiO}_{2}$, and $\sim 6 \%$ each of $\mathrm{K}_{2} \mathrm{O}$ and $\mathrm{Na}_{2} \mathrm{O}$, and very low $\mathrm{TiO}_{2}, \mathrm{MgO}$ and $\mathrm{Fe}_{2} \mathrm{O}_{3}$; $\mathrm{Zr}$ levels of only 40-110 ppm imply significant prior extraction of $\mathrm{Zr}$ from these magmas. No age data are available from these rocks, but the shoshonitic geochemistry and compositional fields on the plots in Figure 10 suggest that they may correlate with highlevel Goonumbla-Wombin-Northparkes shoshonitic intrusions, especially those high- $\mathrm{SiO}_{2}$ intrusions around the Northparkes copper-gold field itself (Lickfold et al. 2007). No REE data are available for these rocks.

\section{Marsden area and E43 intrusives}

Analysed Marsden rocks include monzogabbros, monzodiorites, two quartz monzonites and a monzonite (Table 4). Data plotted on Figure 10, supported by the REE patterns (Figure 11b) for a diorite and a hornblende diorite from Marsden, suggest that most Marsden intrusives are broadly comparable with those from E42 and the Narromine Igneous Complex MMMz. The Marsden rocks we correlate with the regional Copper Hill Suite extend to lower $\mathrm{SiO}_{2}$ compositions than those represented in the Narromine Igneous Complex. However, as for the E42 Muddy Lake Gabbro, data in Figure 10 suggest that some of the more evolved quartz diorite/quartz monzonites may be better correlated with the Copper Hill Suite. Further geochronological and geochemical studies of the Marsden rocks are required to elucidate the compositional groups and relationships represented among the intrusive rocks in this area. However, the E43 diorites have quite flat HREE patterns at significantly higher REE levels than the Copper Hill Suite rocks at similar $\mathrm{SiO}_{2}$ levels, suggesting that they may represent a quite distinct Eastonian medium-K intrusive suite in the Macquarie Arc.

\section{E41 and E42 areas}

E41 samples examined include the most mafic rocks recognised in the Cowal Igneous Complex, these being cumulate wehrlitic (olivine-pyroxene) rocks and olivine-bearing gabbros; three monzodioritic intrusive rocks were also analysed from E41. E42 rocks analysed include mafic diorites transitional to leucogabbro compositions, and several dioritic rocks with $\sim 61-63 \%$ $\mathrm{SiO}_{2}$. Variation diagrams and discrimination plots (Figure 10) show that the more mafic E42 rocks have high-K calc-alkaline to shoshonitic affinities, but since age relationships preclude their correlation with Phase 4 intrusions, our preferred correlation is with the Middle Ordovician Phase 2 Narromine Igneous Complex MMMz suite, and we suggest that an age around 470$465 \mathrm{Ma}$ is likely. Note, however, that several of the monzodiorites analysed from E42 plot within or close to the field for the Copper Hill Suite, implying that more than one intrusion may be present at E42. Further REE data are required to better constrain these relationships. From the vicinity of E42, a monzodiorite from aircore hole 1504/60 at $85.6 \mathrm{~m}$ yielded an initial $\varepsilon_{\mathrm{Nd}}$ value of 7.67 , matching those values for other Middle Ordovician igneous rocks in the Macquarie Arc (see Crawford et al. 2007). 
A. J. Crawford et al.

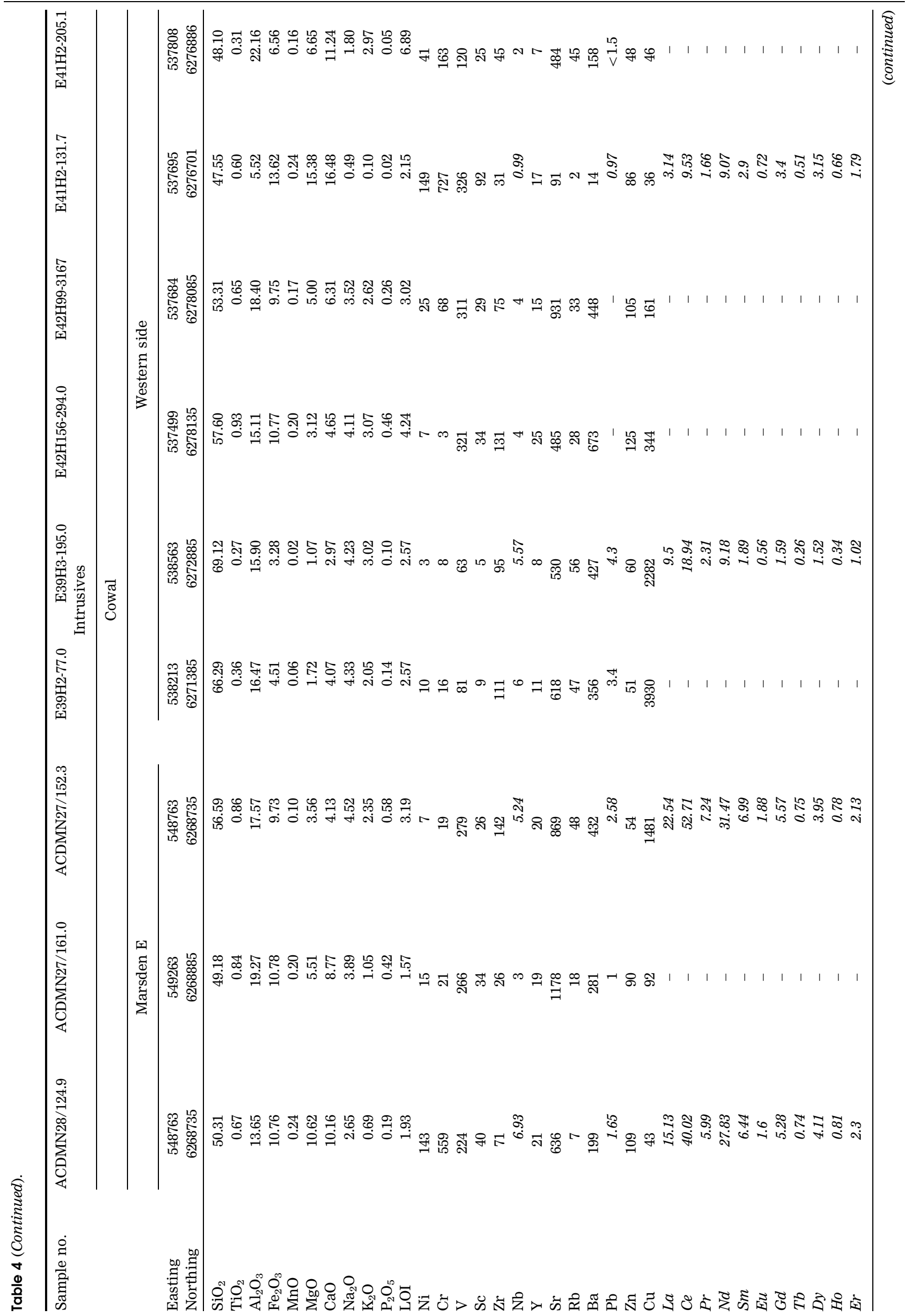




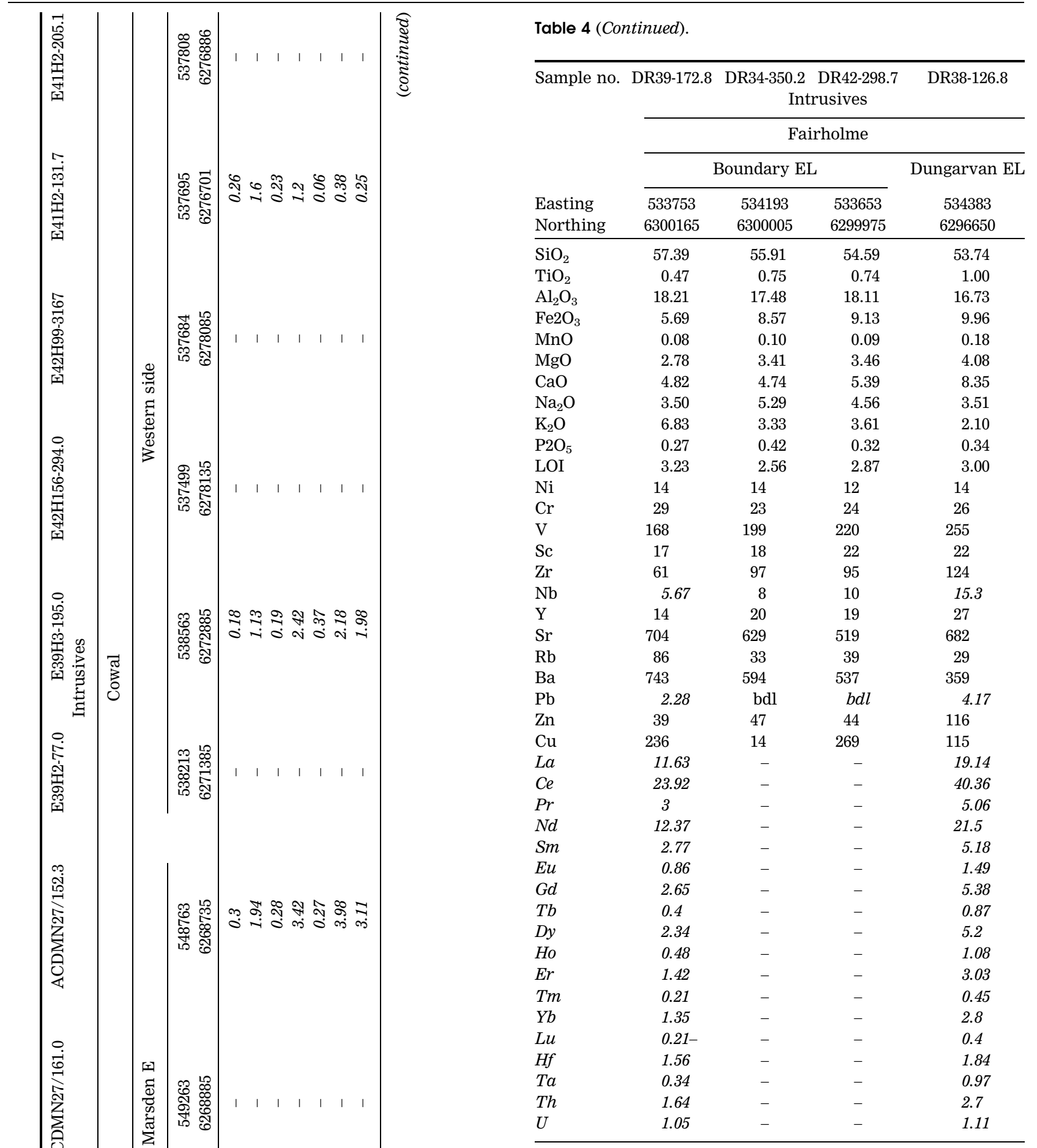

Trace elements in italics measured by ICP-MS, remainder and majors by XRF. bdl, below detection limit. See Appendix 1 for rock types analysed.

E39 area

Rocks drilled around E39 are dominated by medium-K calc-alkaline granodiorites that plot mainly in the fields defined by the Copper Hill Suite on the variation diagrams and discrimination plots in Figures 10. This is in concert with $\mathrm{U}-\mathrm{Pb}$ zircon dates (see above) of $451 \pm$ $4.5 \mathrm{Ma}$ and $447 \pm 7 \mathrm{Ma}$, indicating broadly Eastonian crystallisation ages for these intrusions. The REE pattern (Figure 11a) for an E39 granodiorite matches well those 
for Copper Hill Suite rocks from the Narromine Igneous Complex (and regional Copper Hill Suite rocks).

\section{Fairholme Igneous Complex}

\section{OCCURRENCE AND PETROGRAPHY}

Presumed Ordovician volcanic rocks occur over Newcrest's former Fairholme leases, extending entirely in the subsurface northwest from the north-south trending Boobaroi Shear Zone (Figure 8). Newcrest has carried out regional shallow aircore drilling, and more restricted diamond core drilling of three exploration targets named Dungarvan, Boundary and Gateway, but no geological map of the Fairholme Igneous Complex has been compiled. Most material recovered during Newcrest's extensive aircore program over the Fairholme licence was too weathered for further study, but augite-phyric basaltic lavas were identified from drillholes ACNC162 (1 km north-northeast of Boundary) and ACNC141 (just northeast of Dungarvan). These, and the pillowed and brecciated lavas from Boundary (see below), suggest that the regional Ordovician basement here is dominated by basaltic lavas.

The Boundary prospect was the focus of about 10 diamond drillholes in which fresh rock was encountered mainly 80-90 m downhole. Our logging focussed on drillholes DR39, DR41 and DR42, and showed moderately altered olivine + clinopyroxene-phyric pillow basalts and basaltic breccias. These have been intruded by two distinctly different dyke suites, a transitional alkaline dolerite suite with a maximum dyke thickness of $\sim 20 \mathrm{~m}$, and a suite of massive porphyritic, mainly fine-grained monzodiorites $<1 \mathrm{~m}$ to $>30 \mathrm{~m}$ thick. The latter show significant petrographic variation, extending from microgabbro to almost granodiorite compositions. Most are prominently plagioclase + hornblende-phyric, with subordinate augite sometimes preserved, and ubiquitous small FeTi oxides. Dark transitional alkaline dolerites cut the other rock types, show distinctive pink Ti-augite and are less altered than the other Boundary rocks; they match petrographically similar dykes in the Cowal Igneous Complex, and we suggest that they may be associated with mid-Palaeozoic extensional magmatism in this region.

Dungarvan prospect includes $\sim 12$ diamond drillholes drilled into a northwest-southeast-oriented ovoid dioritic intrusion $\sim 3 \times 1 \mathrm{~km}$ in area. Rocks sampled from holes DR1 and DR38 varied from rather mafic diorites or leucogabbros to very well-preserved cumulate hornblende gabbros. At the third Fairholme prospect, Gateway, drillholes intersected a package of almost mylonitic quartz + sericite + chlorite + pyrite schists with poor or no textural preservation. Bestpreserved samples retain textural evidence that they were originally porphyritic lavas with former phenocrysts of plagioclase and clinopyroxene.

\section{GEOCHEMISTRY}

Lavas

Four analysed pillow lavas and lava breccias from the Boundary prospect (two representative analyses in 

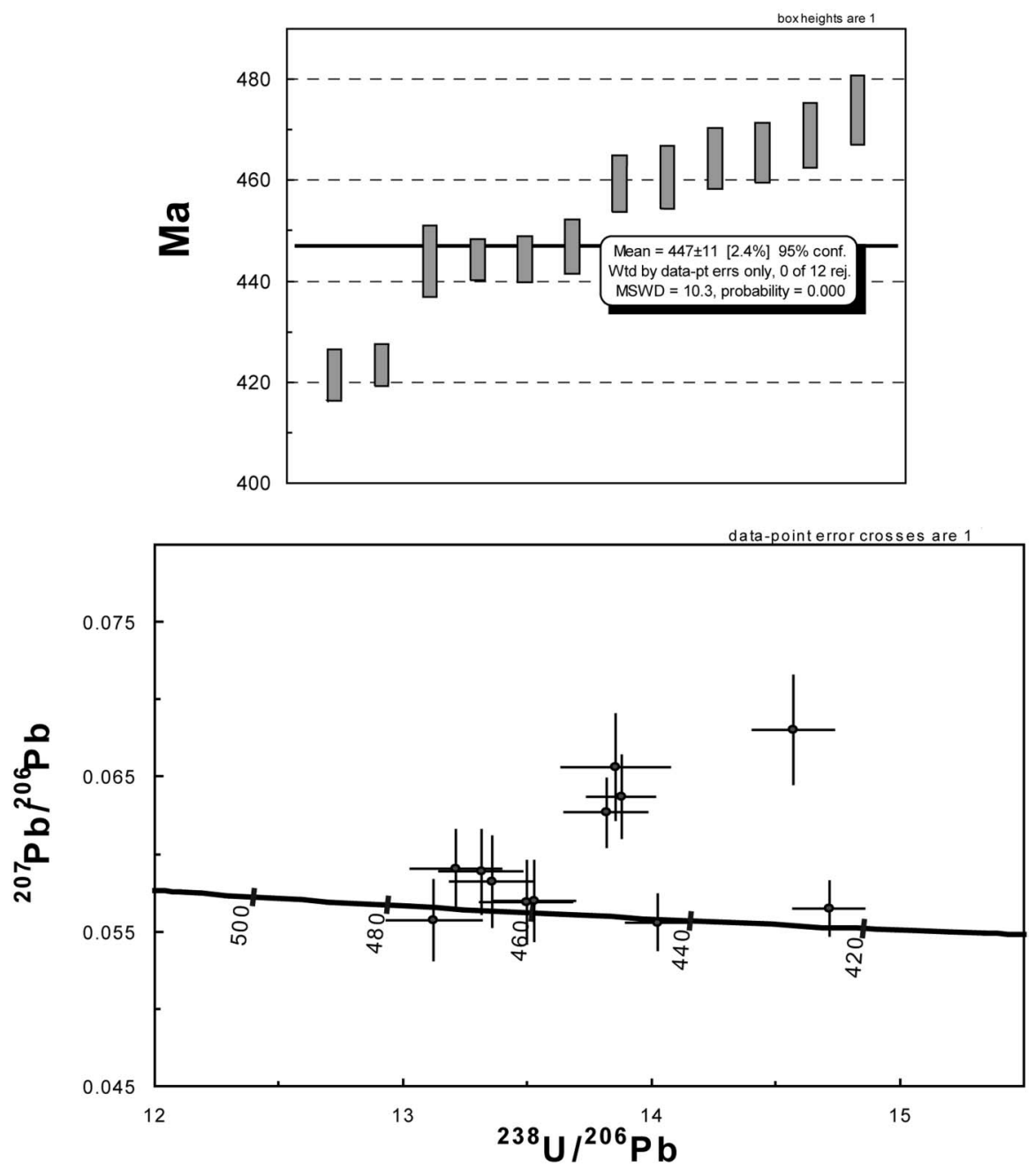

Figure 9 Data for 12 zircons from a granodiorite (drillhole $\mathrm{E} 43 \mathrm{H} 4$ at $159.8 \mathrm{~m}$ ) from the Cowal Igneous Complex, determined by LA ICPMS (methodology given in Meffre et al. 2007). (a) Weighted mean age calculation of the ${ }^{206} \mathrm{~Pb} /{ }^{238} \mathrm{U}$ ages. (b) Tera-Wasserburg concordia plot of the calibrated total ${ }^{238} \mathrm{U} /{ }^{206} \mathrm{~Pb}$ ratio $v$ s the total ${ }^{207} \mathrm{~Pb} /{ }^{206} \mathrm{~Pb}$ ratios. (c) Relative probability plot with stacked histogram of ${ }^{206} \mathrm{~Pb} /{ }^{238} \mathrm{U}$ ages. As for zircons in the Phase 3 (Copper Hill Suite) rocks from the Narromine Igneous Complex, cores of grains shows ages from 474 to $460 \mathrm{Ma}$, whereas rims gives ages averaging around $445 \mathrm{Ma}$.

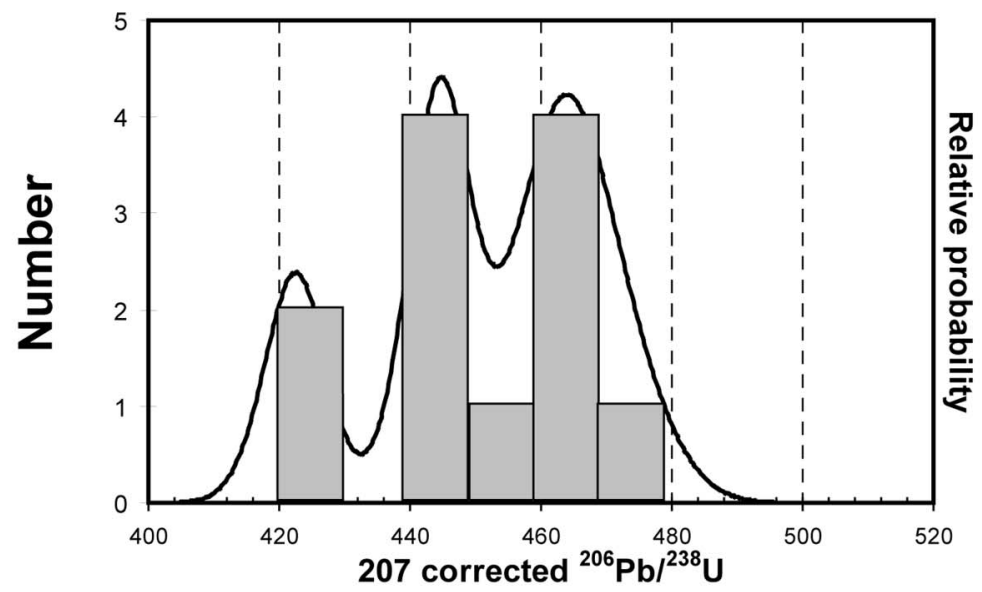

Table 4) define a coherent compositional group similar in most respects to the basaltic end of the Nelungaloo Volcanics range, but extending to higher $\mathrm{MgO}$ and lower $\mathrm{Al}_{2} \mathrm{O}_{3}$ compositions, reflecting the clinopyroxene-rich, lessevolved nature of the Boundary basalts. REE abundences for a Boundary pillow basalt (Figure $4 \mathrm{~b}$ ) define a similarly shaped REE pattern to the Phase 1 Early Ordovician Nelungaloo Volcanics, but with slightly less LREE-enrichment. We conclude that the few mafic volcanics sampled in the Fairholme Igneous Complex are more mafic on average than the Early Ordovician Nelungaloo Volcanics from further north, but with strong compositional similarities and magmatic affinities to the Nelungaloo Volcanics. On this basis, these Fairholme Igneous Complex volcanics are correlated broadly with the Nelungaloo Volcanics. No lavas with affinities to Phase 4 shoshonitic lavas have been recorded to date from the Fairholme Igneous Complex. 

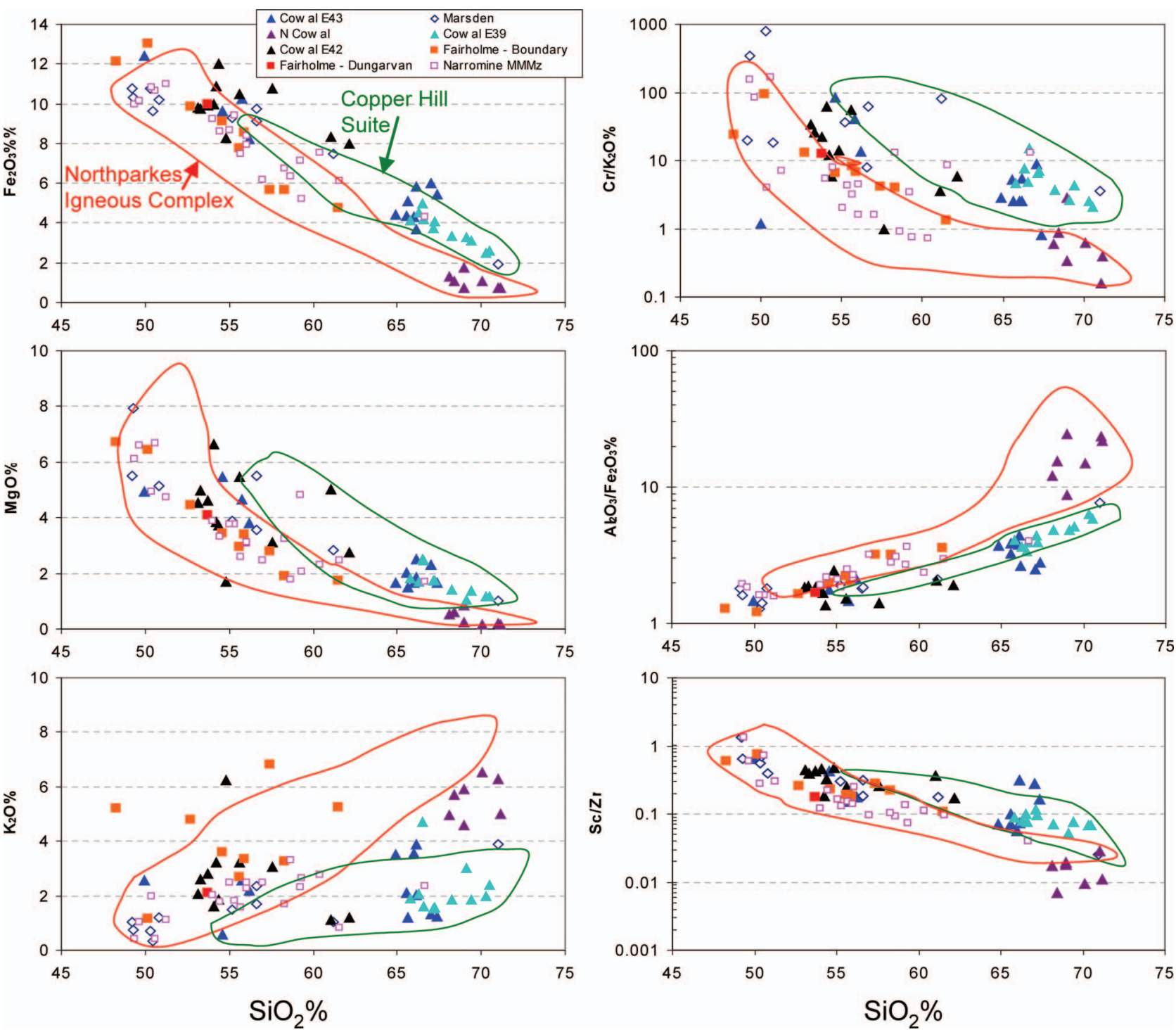

Figure 10 Major- and trace-element variation diagrams for rocks from the Cowal Igneous Complex compared with fields for intrusive rocks from the Northparkes Igneous Complex and Copper Hill Suite (data from Crawford et al. 2007). Also shown are data for MMMz suite intrusives from the Narromine Igneous Complex.

Intrusive rocks

Fairholme intrusives show strong compositional overlap with the shoshonitic Goonumbla-Wombin-Northparkes suite, the only notable difference being that the latter have slightly higher $\mathrm{Zr} / \mathrm{Nb}(\sim 15)$ than the Fairholme rocks $(\sim 10)$, reflecting very slightly higher $\mathrm{Nb}$ in the Fairholme suite. REE patterns for the Dungarvan and Boundary intrusives (Figure 11c) show a strong overlap with the field for Goonumbla-Wombin lavas, lacking the flattening off of the patterns between $\mathrm{La}$ and $\mathrm{Nd}$ that characterises the Boundary basaltic lavas and Nelungaloo-type patterns (Crawford et al. 2007). A hornblende- phyric microdiorite (intrusive latite) dyke from DR34 at Boundary has provided an age-corrected initial $\varepsilon_{\mathrm{Nd}}$ value of +4.74 (Glen et al. 2007a). This value is considerably lower than values for the Cowal Igneous Complex Early Ordovician lavas and Middle Ordovician intrusives, and is within the range of values for the Phase 4 GoonumblaWombin suite shoshonitic magmatism.

In summary, the intrusive rocks at Boundary in particular, but also the Dungarvan diorite, are compositionally shoshonitic, with strong similarities to the Goonumbla and Wombin Volcanics-Northparkes Igneous Complex suites. Given that the Boundary monzodiorites are high-level intrusives into Nelungaloo-

Figure 11 Chondrite-normalised REE patterns for intrusive rocks in the Cowal and Fairholme Igneous Complexes: (a) from prospect E43, compared with Copper Hill Suite intrusive rocks from Narromine, Copper Hill and Cargo; (b) from Marsden, compared with MMMz suite intrusives from the Narromine Igneous Complex; and (c) from the Boundary and Dungarvan prospects in the Fairholme Igneous Complex, showing for comparison a typical Phase 4 shoshonitic andesite from the Goonumbla Volcanics. 


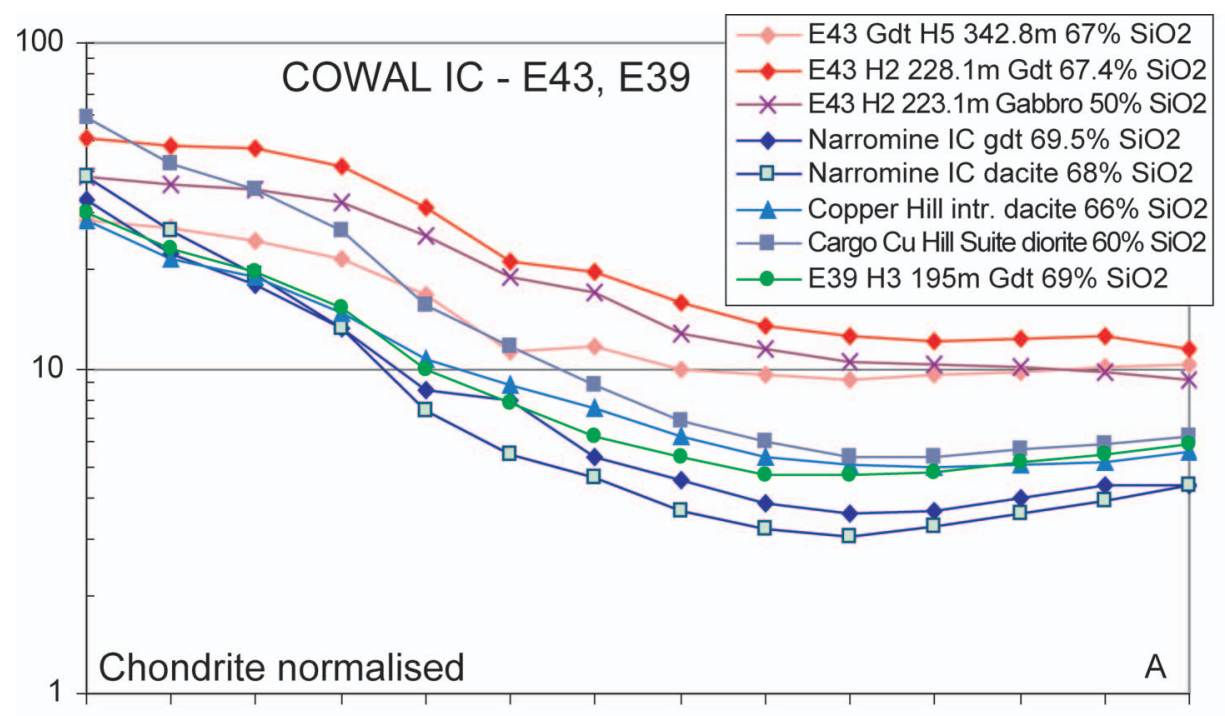

$\begin{array}{lllllllllllllllll}\mathrm{La} & \mathrm{Ce} & \mathrm{Pr} & \mathrm{Nd} & \mathrm{Sm} & \mathrm{Eu} & \mathrm{Gd} & \mathrm{Tb} & \mathrm{Dy} & \mathrm{Ho} & \mathrm{Er} & \mathrm{Tm} & \mathrm{Yb} & \mathrm{Lu}\end{array}$
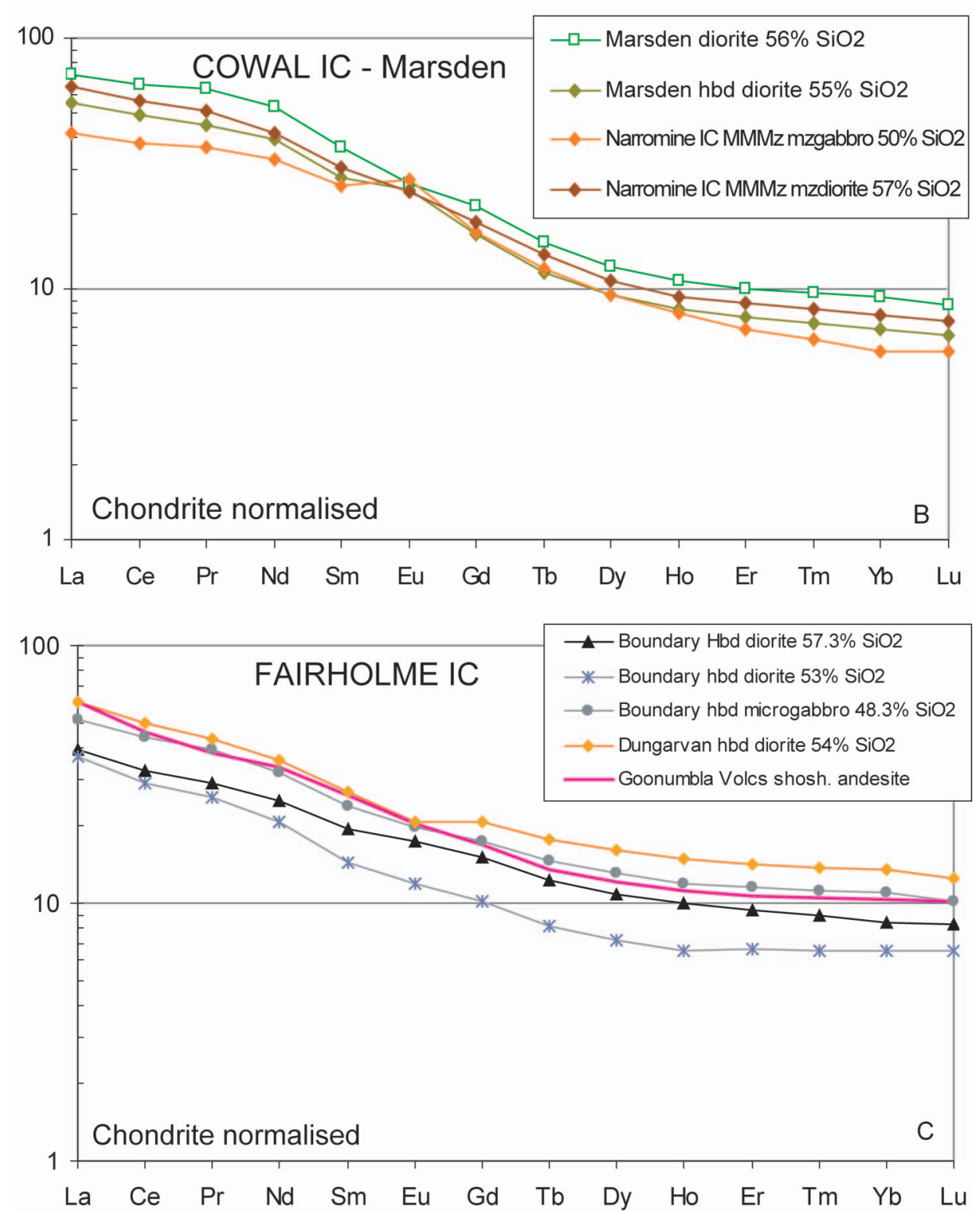
type basaltic lavas, it is difficult to ignore the temporal lithogeochemical pattern similarity to the Parkes region Ordovician suites. On this basis, a Late Ordovician age might be predicted for the Boundary-Dungarvan intrusives.

\section{DISCUSSION}

\section{Origin and significance of the Copper Hill Suite}

Our studies of the distribution, timing and compositional affinities of magmatic rocks in the Macquarie Arc have shown that a widespread but relatively smallvolume magmatic event, which we refer to as the Copper Hill Suite, was emplaced at ca 455-445 Ma, broadly corresponding with the Eastonian interval of limestone deposition and regional uplift (Crawford et al. 2007). Although most voluminous in the Junee-Narromine Volcanic Belt, especially in the Cowal Igneous Complex, high-level intrusive dacites and associated dioritic to granodioritic rocks are known from the Molong Volcanic Belt from the type locality at Copper Hill (Blevin 2002) and further north towards Wellington (Crawford et al. 2007), from the Cargo (Simpson et al. 2007) and Cadia (Squire \& Crawford 2007) areas, and from the Rockley-Gulgong Volcanic Belt from one intrusion on Jenkins Road south of Mudgee (Crawford et al. 2007). Data presented by Blevin (2002) and (Crawford et al. 2007) demonstrate that the Copper Hill Suite has medium-K calc-alkaline affinities, with compositions extending from cumulate hornblende gabbros to granodiorite, although the average composition is probably dioritic. The abundance of hornblende in the cumulate mafic rocks suggests that they probably crystallised from andesitic rather than basaltic magmas.

The compositional range and timing of emplacement of the Copper Hill Suite deserve comment. Evidence from around Molong, and the northern end of the Cargo Block, suggests that emplacement of the stocks of porphyritic dacite and associated more holocrystalline rocks in these areas accompanied significant uplift, with widespread limestone deposition. Similarly, Copper Hill Suite rocks in the Narromine Igneous Complex show a striking textural difference between the typically mediumgrained granodioritic holocrystalline intrusives, and the porphyritic dacites with very fine-grained groundmasses. From drilling, the granodiorite in the western part of the intrusive complex is at least several square kilometres in area, and in NACD8, the granodiorite occurs as a sheet at least $20 \mathrm{~m}$ thick with relatively coarse-grained holocrystalline margins. In contrast, in NACD83, dacite dykes compositionally identical to the granodiorite are at least $10 \mathrm{~m}$ thick with no textural transition from finer grained at the margins to more holocrystalline textures in the central parts of the dyke. The groundmass grainsize of the dacites indicates that these were emplaced at significantly shallower levels than the holocrystalline granodiorites, despite the almost identical geochemical compositions of these rocks. Emplacement during a period of active tectonism and uplift is indicated, and is supported by the absence of extrusive correlates of these rocks, which, if they did exist, were apparently removed by erosion before termination of Macquarie Arc magmatism.

Prior to intrusion of the Copper Hill Suite, Phase 2 magmatism in the Macquarie Arc involved a temporal transition (Crawford et al. 2007) from medium-K (Cargo Volcanics) and mainly high-K calc-alkaline volcanics (lower Fairbridge Volcanics, lower Blayney Volcanics), to basaltic-dominated successions with pronounced shoshonitic compositions (upper Fairbridge Volcanics, Walli Volcanics, Mt Pleasant Basalt Member, Byng Volcanics, upper Blayney Volcanics and Millthorpe Volcanics and correlates in the Rockley-Gulgong Volcanic Belt). The distinctly medium-K calc-alkaline Copper Hill Suite therefore records a departure from this trend, and reversion to magmas with lower-K affinities despite their intermediate $\mathrm{SiO}_{2}$ contents compared with the basaltic compositions dominating the immediately preceding magmatism.

The dominance in the Copper Hill Suite of intermediate and felsic compositions with $\varepsilon_{\mathrm{Nd}}$ values $(>+7$ : Crawford et al. 2007) very close to that of the Ordovician convecting upper mantle, coupled with the apparent absence of basaltic magmas in the petrogenesis of this suite, suggest that its parental magmas may have been generated by partial melting of low-K mafic rocks in the arc basement, or amphibolitic rocks in the subducting slab. Supporting the metamafic melting hypothesis, Girvan (1992) reported the presence of $\mathrm{Mg}-\mathrm{Al}$ chromites with ferritchromite rims at Copper Hill, for which a restitic origin was proposed. Tectonic scenarios accommodating models for the generation of the Copper Hill Suite rocks are given in Glen et al. (2007a).

\section{Zircon inheritance in felsic magmatic systems}

Copper Hill Suite felsic intrusive rocks in both the Narromine and Cowal Intrusive Complexes contain zircon populations that define two distinct age groups. Cores of many grains record ages between 475 and $460 \mathrm{Ma}$, an age range matching that for the regional monzodioritic host-rocks to the Copper Hill Suite granodiorites and dacitic porphyries. Rims of these zircons record mainly Bolindian ages, around 450-441 Ma, taken to be the magmatic age of the Copper Hill Suite magmatism. There is strong evidence, therefore, that zircons from the monzodiorites and related intrusive rocks (in the Narromine Igneous Complex, the Phase $2 \mathrm{MMMz}$ suite) were assimilated by the intruding Copper Hill Suite felsic magmas, and overgrown with Eastonian-age zircon. As zircon solubility is extremely temperature-sensitive, in hotter, more mafic systems, zircon inherited in this manner would be expected to dissolve, leaving little or no evidence of its former existence as xenocrysts (Watson \& Harrison 1983; Miller et al. 2003). The Copper Hill Suite magmas have clear medium-K calc-alkaline affinities, whereas the MMMz monzodiorites and correlates intrusions in the Cowal Igneous Complex show high-K calc-alkaline affinities. Thus, the possibility that the Copper Hill Suite magmas were derived via partial melting of $\mathrm{MMMz}$ suite-type source rocks can be precluded. Thus, the 470-460 Ma zircon cores in Copper Hill Suite rocks cannot be 
regarded as restitic (i.e. representing unmelted components of the source rock), but must have been incorporated locally in the upper crust from the immediate MMMz suite host-rocks to the Copper Hill Suite intrusions. This sounds a note of caution for the interpretation of zircon ages and their significance in I-type granitic magmatic systems.

\section{CONCLUSIONS}

More studies (particularly geochronological) are required to elucidate the time-space relationships of the Macquarie Arc rocks in the unexposed Narromine, Cowal and Fairholme Igneous Complexes. However, our data, considered within the temporal magmatic framework established for the region (Crawford et al. 2007), allow us to draw several important conclusions from this study.

(1) Basaltic to andesitic and trachyandesitic lavas compositionally comparable with the Phase 1 Early Ordovician Nelungaloo Volcanics are present in the Cowal, Fairholme and Narromine Igneous Complexes, and show high-K calc-alkaline compositions with depleted mantle-like $\varepsilon_{\mathrm{Nd}}$ values indicating no interaction of their parental magmas with continental-type crust. By implication, they probably formed in an Early Ordovician intra-oceanic arc.

(2) This volcanic and volcaniclastic basement succession was intruded by large stocks of monzogabbromonzodiorite-monzonite in the Middle Ordovician ( $c a$ 465-460 Ma) that represent Macquarie Arc Phase 2 magmatism.

(3) Stocks and small intrusions representative of the Phase 3 Copper Hill Suite, ranging from hornblende gabbro to granodioritic compositions with medium-K calc-alkaline affinities, and varying texturally from relatively coarse-grained holocrystalline to porphyries with fine-grained groundmasses, were emplaced synchronously with regional-scale uplift between $c a 455$ and $441 \mathrm{Ma}$, mainly in the Bolindian - late Eastonian.

(4) In both the Fairholme Igneous Complex and the northern end of the Cowal Igneous Complex, small mainly felsic intrusions with shoshonitic affinities are interpreted to be correlates of the mainly post-Eastonian Phase 4 felsic intrusions that host porphyry $\mathrm{Cu}-\mathrm{Au}$ mineralisation at Northparkes and Cadia.

\section{ACKNOWLEDGEMENTS}

This work was supported by an ARC SPIRT grant. Considerable assistance in accessing core material was provided by Bruce Mowat and Piers Reynolds (Narromine) and Stuart Smith, John Holliday and Fraser McCorcqudale (Cowal and Fairholme). We acknowledge useful input by Paul McInnes and the staff at Barrick Gold's Cowal exploration office. Phil Robinson, Sarah Gilbert and Katie McGoldrick assisted substantially with obtaining analytical data. Evgeni Bastrakov provided several whole-rock analyses and dates discussed in our study, Dick Glen provided useful comments and editorial assistance, and David Forster and Oliver
Raymond made many useful points in their reviews of this paper.

\section{REFERENCES}

BASTRAKOV E. 1998. Gold mineralisation at the Lake Cowal prospect, New South Wales. PhD thesis, Australian National University, Canberra (unpubl.).

BLEVIN P. L. 2002. The petrographic and compositional character of variably K-enriched magmatic suites associated with Ordovician porphyry $\mathrm{Cu}-\mathrm{Au}$ mineralisation in the Lachlan Fold Belt, Australia. Mineralium Deposita 37, 87-99.

BOYNTON W. V. 1984. Geochemistry of the rare earth elements: meteorite studies. In: Henderson P. ed. Rare Earth Element Geochemistry, pp. 63-114. Elsevier, Amsterdam.

Butera K. M., Williams I. S., Blevin P. L. \& Simpson C. J. 2001. Zircon $\mathrm{U}-\mathrm{Pb}$ dating of Early Palaeozoic monzonitic intrusives from the Goonumbla area, New South Wales. Australian Journal of Earth Sciences 48, 457-464.

Bywater A., Williams S., McInnes P. \& DiJkmans V. 2004. Developments at the Cowal gold project. In: Bierlien F. P. \& Hough M. A. eds. Tectonics to Mineral Discovery - Deconstructing the Lachlan Orogen, pp. 77-82. Geological Society of Australia Abstracts 74.

Cooke D. R., Wilson A. J., House M. J., Wolfe R. C., WAlshe J. L., LICKFOLD V. \& CRAWFORD A. J. 2007. Alkalic porphyry Au-Cu and associated mineral deposits of the Ordovician to Early Silurian Macquarie Arc, New South Wales. Australian Journal of Earth Sciences 54, 445-463.

CraWford A. J., MefFre S., SQUiRe R. J., BARRon L. M. \& FALlOON T. J. 2007. Middle and Late Ordovician magmatic evolution of the Macquarie Arc, Lachlan Orogen, New South Wales. Australian Journal of Earth Sciences 54, 181-214.

Defant M. J. \& DRUMmond M. S. 1990. Derivation of some modern arc magmas by melting of young subducted lithosphere. Nature 347, $662-665$.

Direen N. G., Lyons P., Korsch R. J. \& GLEN R. A. 2001. Integrated geophysical appraisal of crustal architecture in the eastern Lachlan Orogen. Exploration Geophysics 32, 252-262.

Forster D. A., SECCOMBE P. K. \& PHILLIPS D. 2004. Controls on skarn alteration and mineralization at the Cadia deposits, New South Wales, Australia. Economic Geology 99, 761-788.

GIRVAN S. W. 1992. Geology and mineralisation of the Copper Hill porphyry copper-gold deposits near Molong, New South Wales. BSc (Hons) thesis, Australian National University, Canberra, (unpubl.).

Glen R. A., Crawford A. J. \& CoOKe D. R. 2007a. Tectonic setting of porphyry $\mathrm{Cu}-\mathrm{Au}$ mineralisation in the Ordovician Early Silurian Macquarie Arc, Eastern Lachlan Orogen, New South Wales. Australian Journal of Earth Sciences 54, $465-479$.

Glen R. A., Crawford A. J., Percival I. G. \& Barron L. M. 2007b. Early Ordovician development of the Macquarie Arc, Lachlan Orogen, New South Wales. Australian Journal of Earth Sciences 54, $167-179$.

GLen R. A., MefFre S. \& ScotT R. J. 2007c. Benambran Orogeny in the Eastern Lachlan Orogen, Australia. Australian Journal of Earth Sciences 54, 385-415.

Glen R. A., SPencer R., Willmore A., DAvid V. \& ScotT R. J. 2007d. Junee-Narromine Volcanic Belt, Macquarie Arc, Lachlan Orogen, New South Wales: components and structure. Australian Journal of Earth Sciences 54, 215-241.

LickFold V., COOKE D. R., CRAWFord A. J. \& FANNING C. M. 2007. Shoshonitic magmatism and formation of the Northparkes porphyry $\mathrm{Cu}-\mathrm{Au}$ deposits, New South Wales. Australian Journal of Earth Sciences 54, 417-444.

Lickfold V., CoOke D. R., SMith S. G. \& Ullrich T. D. 2003. Endeavour copper-gold porphyry deposits, Northparkes, New South Wales: intrusive history and fluid evolution. Economic Geology 98, 1607-1636.

LyONS P. \& WALLACE D. 1999. Geology and metallogenesis of the Parkes-Grenfell-Wyalong-Condobolin region, New South Wales. Forbes 1:250 000 Geological Sheet and Conference Guide. Australian Geological Survey Organisation Record 1999/20. 
MefFre S., SCott R. J., Glen R. A. \& SQUiRe R. J. 2007. Re-evaluation of contact relationships between Ordovician volcanic belts and the quartz-rich turbidites of the Lachlan Orogen. Australian Journal of Earth Sciences 54, 363-383.

MiLES I. N. \& BRoOKER M. R. 1998. Endeavour 42 deposit, Lake Cowal, New South Wales: a structurally controlled gold deposit. Australian Journal of Earth Sciences 45, 837-847.

Miller C. F., McDowell S. M. \& MAPES R. W. 2003. Hot and cold granites? Implications of zircon saturation temperatures and preservation of inheritance. Geology 31, 529-532.

Percival I. G. \& GLEN R. A. 2007. Ordovician to earliest Silurian history of the Macquarie Arc, Lachlan Orogen, New South Wales. Australian Journal of Earth Sciences 54, 143-165.

PERKINS C., WALSHE J. L. \& MORRISON G. 1995. Metallogenic episodes of the Tasman Fold Belt system, eastern Australia. Economic Geology 90, 1443-1466.

RAYMOND O. L. \& SHERWIN L. 1999. Geology of the Parkes 1:100 000 Geological Sheet 8531, Preliminary Edition. Australian Geological Survey Organisation, Canberra and Geological Survey of New South Wales, Sydney.

SHERWIN L. 1996. Narromine 1:250 000 Geological Sheet SI/55-3. Explanatory Notes. Geological Survey of New South Wales, Sydney.
Simpson C. J., CAS R. A. F. \& ARUNDELL M. C. 2005. Volcanic evolution of a long-lived Ordovician island arc province in the Parkes region of the Lachlan Fold Belt, southeastern Australia. Australian Journal of Earth Sciences 52, 863-886.

Simpson C. J., SCOTT R. J., CRAWFORD A. J. \& MefFre S. 2007. Volcanology, geochemistry and structure of the Ordovician Cargo Volcanics in the Cargo-Walli region, central New South Wales. Australian Journal of Earth Sciences 54, 315-352.

SQUIRE R. J. \& CRAWFORD A. J. 2007. Magmatic characteristics and geochronology of Ordovician igneous rocks from the CadiaNeville region, New South Wales: implications for tectonic evolution. Australian Journal of Earth Sciences 54, 293-314.

Sun S-S. \& MCDonough W. F. 1989. Chemical and isotopic systematics of oceanic basalts: implications for mantle composition and processes. In: Saunders A. D. \& Norry M. J. eds. Magmatism in the Ocean Basins, pp. 313-345. Geological Society of London Special Publication 42.

WATSON E. B. \& HARRISON T. M. 1983. Zircon saturation revisited: temperature and composition effects in a variety of crustal magma types. Earth and Planetary Science Letters 64, 295-304.

Received 1 June 2006; accepted 30 October 2006

\section{APPENDIX 1: ROCK TYPES ANALYSED IN TABLES 1, 3, AND 4}

\begin{tabular}{|c|c|}
\hline Sample no. & Rock type \\
\hline \multicolumn{2}{|c|}{ Narromine Igneous Complex: lavas and dykes (Table 1) } \\
\hline NACD28-80.0 & Moderately plagioclase-phyric andesite lava or narrow dyke \\
\hline NACD96-96.0 & Holocrystalline trachytic textured andesite dyke \\
\hline NACD105-108.4 & Sparsely plagioclase-phyric andesitic dyke \\
\hline NACD137-110.0 & Sericite-chlorite-altered andesitic lava(?) clast \\
\hline NACD20-128.9 & Moderately plagioclase-phyric shallow intrusive andesite \\
\hline \multicolumn{2}{|c|}{ Narromine Igneous Complex: Monzogabbro-Monzodiorite-Monzonite Suite (Table 3) } \\
\hline NACD68-101.4 & Shallow miarolytic monzodiorite \\
\hline NACD82-143.8 & Coarse-grained mafic monzodiorite \\
\hline NACD85-132.7 & Sparsely plagioclase-phyric monzonitic shallow intrusive \\
\hline NACD89-197.0 & Plagioclase-phyric micromonzonite \\
\hline NACD90-144.0 & Greenschist facies monzogabbro \\
\hline NACD90-183.8 & Cumulate monzogabbro with minor altered olivine \\
\hline NACD92-61.2 & Greenschist facies monzogabbro \\
\hline NACD129-134 & Chilled margin monzogabbro \\
\hline NACD105-103.4 & Greenschist facies quartz diorite \\
\hline NACD2-65.0 & Medium-grained monzodiorite \\
\hline NACD96-102.6 & Medium-grained monzonite \\
\hline NACD97-53.0 & Coarse monzogabbro \\
\hline NACD118-55.8 & Medium-grained monzogabbro \\
\hline NACD119-35.7 & Medium-grained monzogabbro \\
\hline NACD119-58.9 & Low greenschist facies monzogabbro \\
\hline NACD121-80.7 & Finer grained gabbro or monzogabbro \\
\hline NACD122-65.8 & Coarse-grained monzodiorite \\
\hline NACD125-64.1 & Coarse-grained hornblende monzogabbro \\
\hline NACD126-60.0 & Medium-grained monzogabbro \\
\hline NACD130-86.9 & Coarser grained monzogabbro \\
\hline NACD131-79.8 & Coarser grained monzogabbro \\
\hline NACD135-97.4 & Slightly altered monzodiorite \\
\hline \multicolumn{2}{|c|}{ Narromine Igneous Complex: Copper Hill Suite (Table 3) } \\
\hline NACD82-120.0 & Shallow intrusive rhyodacite \\
\hline NACD83-116.6 & Plagioclase + quartz + hornblende + FeTiox-phyric dacite \\
\hline NACD83-148.4 & Plagioclase + quartz + hornblende + FeTiox-phyric dacite \\
\hline NACD89-137.2 & Strongly plagioclase-phyric dacite with occasional quartz phenocrysts \\
\hline NACD8-137.7 & Slightly altered granodiorite \\
\hline NACD86-129.1 & Medium-grained granodiorite \\
\hline NACD137-119.1 & Slightly altered quartz diorite \\
\hline
\end{tabular}


APPENDIX 1 (Continued).

Sample no.

Rock type

Cowal and Fairholme Igneous Complexes: Volcanics (Table 4)

E43H5-173.5

E43H4-376.3

E42H103-214.5

E42H361-185.8

DR39-139.8

DR41-94.5

Plagioclase + clinopyroxene + FeTiox + apatite-phyric andesite lava

Formerly glassy lava, altered plagioclase phenocrysts, rare mafics (altered); no apatite

Sparsely plagioclase-phyric glassy trachytic lava, common apatite

Moderately plagioclase-phyric andesite, occasional altered augite, apatite microphenocrysts

Clinopyroxene-phyric vesicular basalt pillowed

Vesicular clinopyroxene + altered olivine-phyric basalt pillow

Cowal and Fairholme Igneous Complexes: Intrusives (Table 4)

LCD12-69.

$1504 / 44-44$

E43H2-228.1

E43H5-342.8

E43H2-337.2

ACDMN28/124.9

ACDMN27/161.0

ACDMN8/152.3

E39H2-77.

E39H3-195.0

E42H156-294.0

E42H99-316

E41H2-131.7

$\mathrm{E} 41 \mathrm{H} 2-205.1$

DR39-172.8

DR34-350.2

DR42-298.7

DR38-126.8

K-feldspar + quartz-phyric shallow intrusive granodiorite

Shallow porphyritic microgranite

Plagioclase-phyric shallow granodiorite

Chilled margin of granodiorite

Holocrystalline hornblende microgabbro, interstitial green biotite

Hornblende gabbro

Hornblende gabbro with plenty of apatite

Diorite

Medium-grained granodiorite

High-level granodiorite

Coarse-grained monzodiorite

Diorite

Cumulate plagioclase wehrlite

Coarse-grained gabbro

Hornblende diorite narrow dyke, no clinopyroxene, fresh hornblende, almost no apatite

Shallow intrusive hornblende + plagioclase-phyric andesite, euhedral hornblendes

Hornblende + plagioclase-phyric shallow intrusive diorite

Hornblende diorite, fresh clinopyroxene, hornblende, biotite 